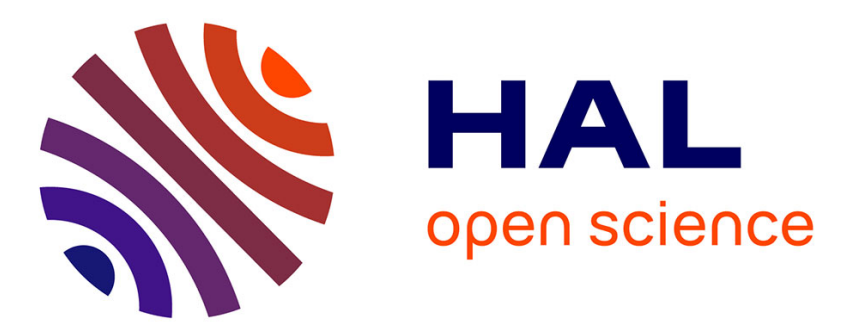

\title{
Wavelet-type expansion of generalized Rosenblatt process and its rate of convergence
}

\author{
Antoine Ayache, Yassine Esmili
}

\section{To cite this version:}

Antoine Ayache, Yassine Esmili. Wavelet-type expansion of generalized Rosenblatt process and its rate of convergence. Journal of Fourier Analysis and Applications, 2020. hal-02307962

\section{HAL Id: hal-02307962 \\ https://hal.science/hal-02307962}

Submitted on 8 Oct 2019

HAL is a multi-disciplinary open access archive for the deposit and dissemination of scientific research documents, whether they are published or not. The documents may come from teaching and research institutions in France or abroad, or from public or private research centers.
L'archive ouverte pluridisciplinaire HAL, est destinée au dépôt et à la diffusion de documents scientifiques de niveau recherche, publiés ou non, émanant des établissements d'enseignement et de recherche français ou étrangers, des laboratoires publics ou privés. 


\title{
Wavelet-type expansion of generalized Rosenblatt process and its rate of convergence
}

\author{
Antoine Ayache and Yassine Esmili \\ UMR CNRS 8524 \\ Laboratoire Paul Painlevé \\ Université de Lille \\ E-mails: antoine.ayache@univ-lille.fr \\ yassine.esmili@univ-lille.fr \\ January 14, 2019
}

\begin{abstract}
Pipiras introduced in the early 2000s an almost surely and uniformly convergent (on compact intervals) wavelet-type expansion of classical Rosenblatt process. Yet, the issue of estimating, almost surely, its uniform rate of convergence remained an open question. The main goal of our present article is to provide an answer to it in the more general framework of generalized Rosenblatt process, under the assumption that the underlying wavelet basis belongs to the class due to Meyer. The main ingredient of our strategy consists in expressing in a non-classical (new) way the approximation errors related with the approximation spaces of a multiresolution analysis of $L^{2}\left(\mathbb{R}^{2}\right)$. Such a non-classical expression may also be of interest in its own right.
\end{abstract}

Running Title: Wavelet expansion of Rosenblatt process revisited.

Key Words. Wiener chaos, self-similar processes, multiresolution analyses, wavelet bases, random series.

AMS Subject Classification (2010). Primary: 60G18, 42C40; secondary: 41 A58.

\section{Introduction and statement of the two main results}

The Rosenblatt process is a non-Gaussian extension of the classical fractional Brownian motion (see e.g. [22, 13]) which was first introduced in the pioneering article [20]. Later, the well-known papers $[23,12,24]$ drew important connections between it and Non-Central 
Limit theorem. Since more than a decade, there has been increasing interest in its study, we refer to the works $[19,1,9,16,25,2,10]$ to cite only a few. More precisely, it is a realvalued non-Gaussian self-similar stochastic process with stationary increments which belongs to the second order Wiener chaos and has continuous paths. We mention in passing that two classical books on Wiener chaoses, multiple Wiener integrals and related topics are [14, 18]. Throughout our article, using the same terminology as in [19], the Rosenblatt process is called fractional Rosenblatt motion (fRm in short) and denoted by $\left\{R_{H}(t)\right\}_{t \in \mathbb{R}_{+}}$since it depends on a parameter $H \in(3 / 4,1)$. Notice that this parameter $H$ is replaced by the parameter $\kappa:=H-1 / 2$ by some authors, because of the fact that $2 \kappa$ corresponds to the self-similarity exponent of fRm. $\left\{R_{H}(t)\right\}_{t \in \mathbb{R}_{+}}$is defined through the following double Wiener integral with respect to a Brownian motion $\{B(x)\}_{x \in \mathbb{R}}$ fixed once and for all:

$$
R_{H}(t):=\int_{\mathbb{R}^{2}}^{\prime} K_{H}\left(t, x_{1}, x_{2}\right) d B\left(x_{1}\right) d B\left(x_{2}\right), \quad \text { for all } t \in \mathbb{R}_{+} .
$$

The symbol $\int_{\mathbb{R}^{2}}^{\prime}$ in (1.1) denotes integration over $\mathbb{R}^{2}$ excluding the diagonal, and the integrand $K_{H}$ is given, for every $\left(t, x_{1}, x_{2}\right) \in \mathbb{R}_{+} \times \mathbb{R}^{2}$, by:

$$
K_{H}\left(t, x_{1}, x_{2}\right):=(\Gamma(H-1 / 2))^{-2} \int_{0}^{t}\left(s-x_{1}\right)_{+}^{H-3 / 2}\left(s-x_{2}\right)_{+}^{H-3 / 2} d s,
$$

with the convention that, for each $(y, \alpha) \in \mathbb{R}^{2}$, one has $y_{+}^{\alpha}:=y^{\alpha}$ if $y$ is positive, and $y_{+}^{\alpha}:=0$ else. Observe that $\Gamma$ in (1.2) is the usual "Gamma function" defined, for every real number $z \in(0,+\infty)$, as $\Gamma(z):=\int_{0}^{+\infty} u^{z-1} e^{-u} d u$. Also, observe that the inequalities $3 / 4<H<1$ imply, for each fixed $t \in \mathbb{R}_{+}$, that the kernel function $\left(x_{1}, x_{2}\right) \mapsto K_{H}\left(t, x_{1}, x_{2}\right)$ belongs to the Hilbert space $L^{2}\left(\mathbb{R}^{2}\right)$; this is why the double Wiener integral in (1.1) is well-defined.

In the early 2000s, a wavelet-type series expansion of $\left\{R_{H}(t)\right\}_{t \in \mathbb{R}_{+}}$, which is almost surely and uniformly convergent in $t$ on any compact interval $I \subset \mathbb{R}_{+}$, was introduced by Pipiras in [19]. Thanks to it, this process can be almost surely approximated, uniformly in $t \in I$, by a sequence of processes $\left(\left\{R_{H, J}(t)\right\}_{t \in \mathbb{R}_{+}}\right)_{J \in \mathbb{N}}$ with continuous paths which are issued from a multiresolution analysis (MRA) of $L^{2}\left(\mathbb{R}^{2}\right)$ and for which efficient simulation methods are available (see $[1,19])$. Yet, the issue of estimating, almost surely, the uniform norm over $I$ of the approximation error, that is $\left\|R_{H}-R_{H, J}\right\|_{I, \infty}:=\sup _{t \in I}\left|R_{H}(t)-R_{H, J}(t)\right|$, remained an open question. The primary motivation behind our article is to provide an answer to it. In order to explain the main focus of our strategy employed to this end, we need to briefly present the fundamental notion of MRA which is the keystone of the wavelet theory. Let us mention that two very classical references on this theory are [11, 17].

A MRA of the Hilbert space $L^{2}\left(\mathbb{R}^{d}\right)$, the integer $d \geq 1$ being arbitrary, is a sequence $\left(V_{j}^{d}\right)_{j \in \mathbb{Z}}$ of closed subspaces of $L^{2}\left(\mathbb{R}^{d}\right)$ satisfying the following four conditions: 
(i) $V_{j}^{d} \subset V_{j+1}^{d}$, for every $j \in \mathbb{Z}$;

(ii) $\bigcap_{j \in \mathbb{Z}} V_{j}^{d}=\{0\}$ and $\bigcup_{j \in \mathbb{Z}} V_{j}^{d}$ is dense in $L^{2}\left(\mathbb{R}^{d}\right)$;

(iii) for any function $f(x) \in L^{2}\left(\mathbb{R}^{d}\right), f(x)$ belongs to $V_{0}^{d}$ if and only if $f\left(2^{j} x\right)$ belongs to $V_{j}^{d}$, for all $j \in \mathbb{Z}$, in other words one has $V_{j}^{d}:=\left\{f\left(2^{j} x\right): f(x) \in V_{0}^{d}\right\}$;

(iv) There exists a function $\phi^{d}(x)$ in $V_{0}^{d}$, called scaling function, such that the sequence $\left\{\phi^{d}(x-l): l \in \mathbb{Z}^{d}\right\}$ forms an orthonormal basis of $V_{0}^{d}$.

Notice that it can easily be derived from (iii) and (iv) that the sequence $\left\{2^{j d / 2} \phi^{d}\left(2^{j} x-l\right)\right.$ : $\left.l \in \mathbb{Z}^{d}\right\}$ is an orthonormal basis of $V_{j}^{d}$, for every fixed $j \in \mathbb{Z}$. The closed subspace $W_{j}^{d}$ of $L^{2}\left(\mathbb{R}^{d}\right)$ denotes the orthogonal complement of $V_{j}^{d}$ in $V_{j+1}^{d}$, that is one has

$$
V_{j+1}^{d}=V_{j}^{d} \stackrel{\perp}{\oplus} W_{j}^{d}, \quad \text { for all } j \in \mathbb{Z} .
$$

Observe that it follows from (1.3) and (ii) that, for any fixed $J \in \mathbb{Z}$, the following three fundamental equalities hold:

$$
V_{J}^{d}=\bigoplus_{-\infty<j<J}^{\perp} W_{j}^{d}
$$

and

$$
L^{2}\left(\mathbb{R}^{d}\right)=V_{J}^{d} \stackrel{\perp}{\oplus}\left(\bigoplus_{J \leq j<+\infty}^{\perp} W_{j}^{d}\right)=\bigoplus_{-\infty<j<+\infty}^{\perp} W_{j}^{d} .
$$

It is known that there are $2^{d}-1$ functions $\psi^{d, p}, p \in\left\{1, \ldots, 2^{d}-1\right\}$, belonging to $W_{0}^{d}$, called mother wavelets, such that the sequence $\left\{\psi^{d, p}(x-k):(p, k) \in\left\{1, \ldots, 2^{d}-1\right\} \times\right.$ $\left.\mathbb{Z}^{d}\right\}$ is an orthonormal basis of $W_{0}^{d}$. A straightforward consequence is that the sequence $\left\{2^{j d / 2} \psi^{d, p}\left(2^{j} x-k\right):(p, k) \in\left\{1, \ldots, 2^{d}-1\right\} \times \mathbb{Z}^{d}\right\}$ is an orthonormal basis of $W_{j}^{d}$, for every fixed $j \in \mathbb{Z}$. Thus, using (1.4) and (1.5), it can be shown that:

Theorem 1.1 (see e.g. $[\mathbf{1 7}, \mathbf{1 1}]$ ) Assume that $J \in \mathbb{Z}$ is arbitrary and fixed.

1. Not only the sequence $\left\{2^{J d / 2} \phi^{d}\left(2^{J} x-l\right): l \in \mathbb{Z}^{d}\right\}$ is an orthonormal basis of $V_{J}^{d}$ but also the sequence

$$
\left\{2^{J d / 2} \psi^{d, p}\left(2^{J} x-k\right):(p, j, k) \in\left\{1, \ldots, 2^{d}-1\right\} \times \mathbb{Z} \times \mathbb{Z}^{d} \text { and } j<J\right\} .
$$

2. The sequences

$$
\begin{aligned}
& \left\{2^{J d / 2} \phi^{d}\left(2^{J} x-l\right): l \in \mathbb{Z}^{d}\right\} \\
& \cup\left\{2^{j d / 2} \psi^{d, p}\left(2^{j} x-k\right):(p, j, k) \in\left\{1, \ldots, 2^{d}-1\right\} \times \mathbb{Z} \times \mathbb{Z}^{d} \text { and } j \geq J\right\}
\end{aligned}
$$


and

$$
\left\{2^{j d / 2} \psi^{d, p}\left(2^{j} x-k\right):(p, j, k) \in\left\{1, \ldots, 2^{d}-1\right\} \times \mathbb{Z} \times \mathbb{Z}^{d}\right\}
$$

are two orthonormal bases of $L^{2}\left(\mathbb{R}^{d}\right)$. Such bases of $L^{2}\left(\mathbb{R}^{d}\right)$ are called wavelet bases.

Usually, for any $d \geq 2$, a MRA $\left(V_{j}^{d}\right)_{j \in \mathbb{Z}}$ of $L^{2}\left(\mathbb{R}^{d}\right)$ is obtained starting from a MRA $\left(V_{j}^{1}\right)_{j \in \mathbb{Z}}$ of $L^{2}(\mathbb{R})$ by making use of the tensor product method. It is important for our purposes to briefly describe this method in the case where $d=2$. In all the sequel, for the sake of simplicity, $\left(V_{j}^{1}\right)_{j \in \mathbb{Z}}$ is denoted by $\left(V_{j}\right)_{j \in \mathbb{Z}}$ and corresponding scaling function and mother wavelet are denoted by $\phi$ and $\psi$. Throughout our article, these two functions are assumed to be real-valued. The tensor product method simply consists in defining, for every $j \in \mathbb{Z}$, the space $V_{j}^{2}$ as the tensor product:

$$
V_{j}^{2}:=V_{j} \otimes V_{j}
$$

In other words, $V_{j}^{2}$ is the closed subspace of $L^{2}\left(\mathbb{R}^{2}\right)$ spanned by the set of functions $\left\{g\left(x_{1}\right) h\left(x_{2}\right)\right.$, $g \in V_{j}$ and $\left.h \in V_{j}\right\}$. Then a scaling function associated to $\left(V_{j}^{2}\right)_{j \in \mathbb{Z}}$ is $\left(x_{1}, x_{2}\right) \mapsto \phi\left(x_{1}\right) \phi\left(x_{2}\right)$, and three corresponding mother wavelets are $\left(x_{1}, x_{2}\right) \mapsto \phi\left(x_{1}\right) \psi\left(x_{2}\right),\left(x_{1}, x_{2}\right) \mapsto \psi\left(x_{1}\right) \phi\left(x_{2}\right)$ and $\left(x_{1}, x_{2}\right) \mapsto \psi\left(x_{1}\right) \psi\left(x_{2}\right)$. Such a MRA $\left(V_{j}^{2}\right)_{j \in \mathbb{Z}}$ was implicitly used in Section 4 of [19] in order to construct the stochastic processes $\left\{R_{H, J}(t)\right\}_{t \in \mathbb{R}_{+}}, J \in \mathbb{N}$, which approximate the fRm $\left\{R_{H}(t)\right\}_{t \in \mathbb{R}_{+}}$. More precisely, for any fixed $t \in \mathbb{R}_{+}$, the functions $\left(x_{1}, x_{2}\right) \mapsto K_{H, J}\left(t, x_{1}, x_{2}\right)$ and $\left(x_{1}, x_{2}\right) \mapsto K_{H, J}^{\perp}\left(t, x_{1}, x_{2}\right)$ respectively denote the orthogonal projections in $L^{2}\left(\mathbb{R}^{2}\right)$ of the kernel function $\left(x_{1}, x_{2}\right) \mapsto K_{H}\left(t, x_{1}, x_{2}\right)$ (see (1.2)) respectively on the space $V_{J}^{2}$ and on its orthogonal complement $\left(V_{J}^{2}\right)^{\perp}$. The random variable $R_{H, J}(t)$ is defined as:

$$
R_{H, J}(t):=\int_{\mathbb{R}^{2}}^{\prime} K_{H, J}\left(t, x_{1}, x_{2}\right) d B\left(x_{1}\right) d B\left(x_{2}\right) .
$$

Observe that one can derive from (1.1) and (1.7) that

$$
R_{H}(t)-R_{H, J}(t):=\int_{\mathbb{R}^{2}}^{\prime} K_{H, J}^{\perp}\left(t, x_{1}, x_{2}\right) d B\left(x_{1}\right) d B\left(x_{2}\right) .
$$

In order to estimate almost surely the approximation error $\left\|R_{H}-R_{H, J}\right\|_{I, \infty}$, it is crucial to express $R_{H}(t)-R_{H, J}(t)$ in a convenient way. Under some general conditions on $\phi$ and $\psi$, an expression for it as a random series is given in the statement of Theorem 1 in [19]. Basically, this expression for $R_{H}(t)-R_{H, J}(t)$ is issued from the expansion of the function $\left(x_{1}, x_{2}\right) \mapsto K_{H, J}^{\perp}\left(t, x_{1}, x_{2}\right)$ in the classical orthonormal basis of $\left(V_{J}^{2}\right)^{\perp}$, namely:

$$
\begin{aligned}
\left\{2^{j} \phi\left(2^{j} x_{1}-k_{1}\right) \psi\left(2^{j} x_{2}-k_{2}\right),\right. & 2^{j} \psi\left(2^{j} x_{1}-k_{1}\right) \phi\left(2^{j} x_{2}-k_{2}\right), \\
& \left.2^{j} \psi\left(2^{j} x_{1}-k_{1}\right) \psi\left(2^{j} x_{2}-k_{2}\right):\left(j, k_{1}, k_{2}\right) \in \mathbb{Z}^{3} \text { and } j \geq J\right\} .
\end{aligned}
$$


Unfortunately, this expression has a drawback: the terms of the random series providing it, that is the random variables $Z_{\kappa, d}^{(j)}(t), j \geq J$ defined in relation (4.9) of [19], are correlated. In order to avoid such a drawback, our strategy consists to expand the function $\left(x_{1}, x_{2}\right) \mapsto$ $K_{H, J}^{\perp}\left(t, x_{1}, x_{2}\right)$ in another much less classical orthonormal basis of $\left(V_{J}^{2}\right)^{\perp}$, namely:

$\mathcal{B}_{J}^{\prime \prime}:=\left\{2^{\frac{j_{1}+j_{2}}{2}} \psi\left(2^{j_{1}} x_{1}-k_{1}\right) \psi\left(2^{j_{2}} x_{2}-k_{2}\right):\left(j_{1}, j_{2}, k_{1}, k_{2}\right) \in \mathbb{Z}^{4}\right.$ and $\left.j_{1} \vee j_{2}:=\max \left\{j_{1}, j_{2}\right\} \geq J\right\}$.

We mention in passing that the fact that $\mathcal{B}_{J}^{\prime \prime}$ is an orthonormal basis of $\left(V_{J}^{2}\right)^{\perp}$ can be shown in the following way. The first part of Theorem 1.1 (with $d=1$ ) and (1.6) imply that

$$
\mathcal{B}_{J}^{\prime}:=\left\{2^{\frac{j_{1}+j_{2}}{2}} \psi\left(2^{j_{1}} x_{1}-k_{1}\right) \psi\left(2^{j_{2}} x_{2}-k_{2}\right):\left(j_{1}, j_{2}, k_{1}, k_{2}\right) \in \mathbb{Z}^{4} \text { and } j_{1} \vee j_{2}<J\right\}
$$

is an orthonormal basis of $V_{J}^{2}$. Moreover, the second part of Theorem 1.1 (with $d=1$ ) and (1.6) entail that

$$
\mathcal{B}:=\left\{2^{\frac{j_{1}+j_{2}}{2}} \psi\left(2^{j_{1}} x_{1}-k_{1}\right) \psi\left(2^{j_{2}} x_{2}-k_{2}\right):\left(j_{1}, j_{2}, k_{1}, k_{2}\right) \in \mathbb{Z}^{4}\right\}
$$

is an orthonormal basis of $L^{2}\left(\mathbb{R}^{2}\right)$. Combining these two results with the equality $L^{2}\left(\mathbb{R}^{2}\right)=$ $V_{J}^{2} \stackrel{\perp}{\oplus}\left(V_{J}^{2}\right)^{\perp}$, it follows that $\mathcal{B}_{J}^{\prime \prime}$ is an orthonormal basis of $\left(V_{J}^{2}\right)^{\perp}$.

Our strategy also works in the more general framework of the generalized Rosenblatt process, that we call generalized fractional Rosenblatt motion (gfRm). It was first introduced by Maejima and Tudor in their paper [15]. In the last few years, several articles related to it, written by Bai and Taqqu, were published (see $[4,5,6,7,8]$ ). The gfRm depends on two parameters $H_{1}$ and $H_{2}$ satisfying

$$
H_{1}, H_{2} \in(1 / 2,1) \text { and } H_{1}+H_{2}>3 / 2 \text {. }
$$

It is denoted by $\left\{R_{H_{1}, H_{2}}(t)\right\}_{t \in \mathbb{R}_{+}}$and defined as:

$$
R_{H_{1}, H_{2}}(t):=\int_{\mathbb{R}^{2}}^{\prime} K_{H_{1}, H_{2}}\left(t, x_{1}, x_{2}\right) d B\left(x_{1}\right) d B\left(x_{2}\right), \quad \text { for all } t \in \mathbb{R}_{+},
$$

where, the integrand $K_{H_{1}, H_{2}}$ is given, for every $\left(t, x_{1}, x_{2}\right) \in \mathbb{R}_{+} \times \mathbb{R}^{2}$, by:

$$
K_{H_{1}, H_{2}}\left(t, x_{1}, x_{2}\right):=\frac{1}{\Gamma\left(H_{1}-1 / 2\right) \Gamma\left(H_{2}-1 / 2\right)} \int_{0}^{t}\left(s-x_{1}\right)_{+}^{H_{1}-3 / 2}\left(s-x_{2}\right)_{+}^{H_{2}-3 / 2} d s .
$$

Notice that the condition (1.11) implies that, for each fixed $t \in \mathbb{R}_{+}$, the kernel function $\left(x_{1}, x_{2}\right) \mapsto K_{H_{1}, H_{2}}\left(t, x_{1}, x_{2}\right)$ is in $L^{2}\left(\mathbb{R}^{2}\right)$, which guarantees the existence of the double Wiener integral in (1.12). Also notice that the gfRm reduces to the fRm when $H_{1}=H_{2}$. Similarly to the fRm, the gfRm belongs to the second order Wiener chaos, it has continuous paths, and 
it is self-similar with stationary increments; the self-similarity exponent being the quantity $H_{1}+H_{2}-1$. For any fixed $J \in \mathbb{N}$ and $t \in \mathbb{R}_{+}$, the functions $\left(x_{1}, x_{2}\right) \mapsto K_{H_{1}, H_{2}, J}\left(t, x_{1}, x_{2}\right)$ and $\left(x_{1}, x_{2}\right) \mapsto K_{H_{1}, H_{2}, J}^{\perp}\left(t, x_{1}, x_{2}\right)$ are defined in the same way as $\left(x_{1}, x_{2}\right) \mapsto K_{H, J}\left(t, x_{1}, x_{2}\right)$ and $\left(x_{1}, x_{2}\right) \mapsto K_{H, J}^{\perp}\left(t, x_{1}, x_{2}\right)$. Namely, they respectively are the orthogonal projections in $L^{2}\left(\mathbb{R}^{2}\right)$ of the kernel function $\left(x_{1}, x_{2}\right) \mapsto K_{H_{1}, H_{2}}\left(t, x_{1}, x_{2}\right)$ (see (1.13)) respectively on the space $V_{J}^{2}$ and on its orthogonal complement $\left(V_{J}^{2}\right)^{\perp}$. Similarly to (1.7) and (1.8), the random variable $R_{H_{1}, H_{2}, J}(t)$ is defined as:

$$
R_{H_{1}, H_{2}, J}(t):=\int_{\mathbb{R}^{2}}^{\prime} K_{H_{1}, H_{2}, J}\left(t, x_{1}, x_{2}\right) d B\left(x_{1}\right) d B\left(x_{2}\right),
$$

and one can derive from (1.12) and (1.14) that

$$
R_{H_{1}, H_{2}}(t)-R_{H_{1}, H_{2}, J}(t):=\int_{\mathbb{R}^{2}}^{\prime} K_{H_{1}, H_{2}, J}^{\perp}\left(t, x_{1}, x_{2}\right) d B\left(x_{1}\right) d B\left(x_{2}\right) .
$$

We mention in passing that it results from the isometry property of double Wiener integral and from the Kolmogorov's continuity theorem that, for any fixed $J \in \mathbb{N}$, the stochastic processes $\left\{R_{H_{1}, H_{2}, J}(t)\right\}_{t \in \mathbb{R}_{+}}$and $\left\{R_{H_{1}, H_{2}}(t)-R_{H_{1}, H_{2}, J}(t)\right\}_{t \in \mathbb{R}_{+}}$have continuous paths.

By expanding, for each fixed $J \in \mathbb{N}$ and $t \in \mathbb{R}$, the function $\left(x_{1}, x_{2}\right) \mapsto K_{H_{1}, H_{2}, J}^{\perp}\left(t, x_{1}, x_{2}\right)$ in the basis $\mathcal{B}_{J}^{\prime \prime}$ (see (1.9)), and by using (1.15) as well as the isometry property of double Wiener integral, one obtains that

$$
R_{H_{1}, H_{2}}(t)-R_{H_{1}, H_{2}, J}(t)=\sum_{\left(j_{1}, j_{2}\right) \in \mathbb{Z}^{2}, j_{1} \vee j_{2} \geq J} \sum_{\left(k_{1}, k_{2}\right) \in \mathbb{Z}^{2}} \mathcal{K}_{j_{1}, j_{2}}^{k_{1}, k_{2}}(t) \varepsilon_{j_{1}, j_{2}}^{k_{1}, k_{2}},
$$

Notice that, for all $\left(j_{1}, j_{2}, k_{1}, k_{2}\right) \in \mathbb{Z}^{4}$, the real-valued deterministic coefficient $\mathcal{K}_{j_{1}, j_{2}}^{k_{1}, k_{2}}(t)$ is given by:

$$
\mathcal{K}_{j_{1}, j_{2}}^{k_{1}, k_{2}}(t):=2^{\frac{j_{1}+j_{2}}{2}} \int_{\mathbb{R}^{2}} K_{H_{1}, H_{2}}\left(t, x_{1}, x_{2}\right) \psi\left(2^{j_{1}} x_{1}-k_{1}\right) \psi\left(2^{j_{2}} x_{2}-k_{2}\right) d x_{1} d x_{2} ;
$$

we mention that $(1.13),(1.17)$ and the dominated convergence theorem imply that $\mathcal{K}_{j_{1}, j_{2}}^{k_{1}, k_{2}}$ is a continuous function on $\mathbb{R}_{+}$. Also notice that $\varepsilon_{j_{1}, j_{2}}^{k_{1}, k_{2}}$ is the random variable of the second order Wiener chaos defined, for all $\left(j_{1}, j_{2}, k_{1}, k_{2}\right) \in \mathbb{Z}^{4}$, as:

$$
\varepsilon_{j_{1}, j_{2}}^{k_{1}, k_{2}}:=2^{\frac{j_{1}+j_{2}}{2}} \int_{\mathbb{R}^{2}}^{\prime} \psi\left(2^{j_{1}} x_{1}-k_{1}\right) \psi\left(2^{j_{2}} x_{2}-k_{2}\right) d B\left(x_{1}\right) d B\left(x_{2}\right) .
$$

Observe that our previous arguments for deriving the equality (1.16) only allow to assert that the random series in it is, for each fixed $t \in \mathbb{R}_{+}$and $J \in \mathbb{N}$, unconditionally convergent in $L^{2}(\Omega)$, where $\Omega$ denotes the underlying probability space. Yet, in the proof of our 
Theorem 1.2, stated below, we show that this random series is also almost surely convergent uniformly in $t \in I$. Let us point out that one may assume without any restriction that the latter compact interval is of the form $I=[0, T]$, where the real number $T>2$ is arbitrary and fixed. This assumption is systematically made from now on.

The goal of Section 2 of our article is to derive the following theorem which provides an almost sure estimate of the approximation error $\left\|R_{H_{1}, H_{2}}-R_{H_{1}, H_{2}, J}\right\|_{I, \infty}$.

Theorem 1.2 Assume that $\psi$ in (1.9) is a Meyer's mother wavelet; that is $\psi$ belongs to the Schwartz class $S(\mathbb{R})$ and its Fourier transform $\widehat{\psi}$ is compactly supported with support satisfying

$$
\operatorname{supp} \widehat{\psi} \subseteq\left\{\xi \in \mathbb{R}: \quad \frac{2 \pi}{3} \leq|\xi| \leq \frac{8 \pi}{3}\right\} .
$$

Then, for all compact interval $I \subset \mathbb{R}_{+}$, there exists an almost surely finite random variable $C$ (depending on I) for which one has, almost surely, for each $J \in \mathbb{N}$,

$$
\left\|R_{H_{1}, H_{2}}-R_{H_{1}, H_{2}, J}\right\|_{I, \infty}:=\sup _{t \in I}\left|R_{H_{1}, H_{2}}(t)-R_{H_{1}, H_{2}, J}(t)\right| \leq C J 2^{-J\left(H_{1}+H_{2}-3 / 2\right)} .
$$

Let us now present the goal of Section 3 of our article. Using (1.12) and the wavelet basis $\mathcal{B}$ (see (1.10)), similarly to (1.16) one can derive, for each fixed $t \in \mathbb{R}_{+}$, that

$$
R_{H_{1}, H_{2}}(t)=\sum_{\left(j_{1}, j_{2}, k_{1}, k_{2}\right) \in \mathbb{Z}^{4}} \mathcal{K}_{j_{1}, j_{2}}^{k_{1}, k_{2}}(t) \varepsilon_{j_{1}, j_{2}}^{k_{1}, k_{2}}
$$

where the random series is unconditionally convergent in $L^{2}(\Omega)$. The main goal of Section 3 is to show that it is also convergent in a much stronger sense: almost surely, uniformly in $t \in I:=[0, T]$, and jointly in the four indices $j_{1}, j_{2}, k_{1}$ and $k_{2}$; and to obtain an estimate of the almost sure rate of convergence. More precisely, the following theorem is derived in Section 3:

Theorem 1.3 The four real numbers $T>2$ and $b, d, g \in(0,+\infty)$ are arbitrary and fixed. For all $t \in \mathbb{R}_{+}$and $n \in \mathbb{N}$, let $\widetilde{R}_{H_{1}, H_{2}, n}(t)$ be the random variable of the second order Wiener chaos defined as the finite random sum:

$$
\widetilde{R}_{H_{1}, H_{2}, n}(t):=\sum_{\left(j_{1}, j_{2}, k_{1}, k_{2}\right) \in \mathcal{S}_{n}^{+} \cup \mathcal{S}_{n}^{-}} \mathcal{K}_{j_{1}, j_{2}}^{k_{1}, k_{2}}(t) \varepsilon_{j_{1}, j_{2}}^{k_{1}, k_{2}},
$$

where $\mathcal{S}_{n}^{+}$and $\mathcal{S}_{n}^{-}$are the two finite disjoint sets such that

$$
\mathcal{S}_{n}^{+}:=\left\{\left(j_{1}, j_{2}, k_{1}, k_{2}\right) \in \mathbb{Z}^{4}:-2^{n b} \leq j_{1} \wedge j_{2}, 0 \leq j_{1} \vee j_{2}<n,\left|k_{1}\right| \leq 2^{n+1} T,\left|k_{2}\right| \leq 2^{n+1} T\right\}
$$


and

$$
\mathcal{S}_{n}^{-}:=\left\{\left(j_{1}, j_{2}, k_{1}, k_{2}\right) \in \mathbb{Z}^{4}:-2^{n d} \leq j_{1} \wedge j_{2} \leq j_{1} \vee j_{2}<0,\left|k_{1}\right| \leq 2^{n g},\left|k_{2}\right| \leq 2^{n g}\right\} ;
$$

recall that $j_{1} \wedge j_{2}:=\min \left\{j_{1}, j_{2}\right\}$ and $j_{1} \vee j_{2}:=\max \left\{j_{1}, j_{2}\right\}$. Then, under the assumption that $\psi$ in (1.10) is a Meyer's mother wavelet, there exists an almost surely finite random variable $C$ (depending on $T, b, d, g$ ) for which one has, almost surely, for each $n \in \mathbb{N}$,

$$
\left\|R_{H_{1}, H_{2}}-\widetilde{R}_{H_{1}, H_{2}, n}\right\|_{I, \infty}:=\sup _{t \in I}\left|R_{H_{1}, H_{2}}(t)-\widetilde{R}_{H_{1}, H_{2}, n}(t)\right| \leq C n 2^{-n\left(H_{1}+H_{2}-3 / 2\right)},
$$

where $I:=[0, T]$.

\section{Proof of Theorem 1.2}

In order to prove Theorem 1.2 one needs to obtain several intermediary results.

First one shows that, for all $t \in \mathbb{R}_{+}$and $\left(j_{1}, j_{2}, k_{1}, k_{2}\right) \in \mathbb{Z}^{4}$, the deterministic coefficient $\mathcal{K}_{j_{1}, j_{2}}^{k_{1}, k_{2}}(t)$ (see (1.17)) can be nicely expressed in terms of $\Psi_{H_{1}}$ and $\Psi_{H_{2}}$, the two left-sided fractional primitives of orders $H_{1}-1 / 2$ and $H_{2}-1 / 2$ of the Meyer's mother wavelet $\psi$. One refers to the book [21] for a classical reference on the fundamental notions of fractional primitives and derivatives. Recall that, for any $H \in(1 / 2,1)$, the left-sided fractional primitive of $\psi$ of order $H-1 / 2$ is the real-valued function, we denote by $\Psi_{H}$, defined as:

$$
\Psi_{H}(s):=\frac{1}{\Gamma(H-1 / 2)} \int_{\mathbb{R}}(s-y)_{+}^{H-3 / 2} \psi(y) d y, \quad \text { for all } s \in \mathbb{R} .
$$

It inherits some important properties of the Meyer's mother wavelet $\psi$, namely:

Remark 2.1 The function $\Psi_{H}$ belongs to the Schwartz class $S(\mathbb{R})$. This means that it is infinitely differentiable on the real line, and that itself and its derivative of any order have rapid decrease at infinity; in other words, for each fixed (positive) real number $L$, one has

$$
\sup _{x \in \mathbb{R}}\left\{(3+|x|)^{L}\left|\Psi_{H}(x)\right|\right\}<+\infty,
$$

and (2.2) remains valid when $\Psi_{H}$ is replaced by its derivative of an arbitrary order. Moreover, its Fourier transform $\widehat{\Psi}_{H}$ is the infinitely differentiable function on the real line given by:

$$
\widehat{\Psi}_{H}(0)=0 \quad \text { and } \quad \widehat{\Psi}_{H}(\xi)=(i \xi)^{-(H-1 / 2)} \widehat{\psi}(\xi), \quad \text { for every } \xi \in \mathbb{R} \backslash\{0\} .
$$

Thus, in view of (1.19), $\widehat{\Psi}_{H}$ has a compact support satisfying:

$$
\operatorname{supp} \widehat{\Psi}_{H} \subseteq\left\{\xi \in \mathbb{R}: \quad \frac{2 \pi}{3} \leq|\xi| \leq \frac{8 \pi}{3}\right\} .
$$


The proof of Remark 2.1 has been skipped since it is very classical. The following remark shows that the deterministic coefficient $\mathcal{K}_{j_{1}, j_{2}}^{k_{1}, k_{2}}(t)$ can be nicely expressed in terms of the functions $\Psi_{H_{1}}$ and $\Psi_{H_{2}}$.

Remark 2.2 Let $t \in \mathbb{R}_{+}$and $\left(j_{1}, j_{2}, k_{1}, k_{2}\right) \in \mathbb{Z}^{4}$ be arbitrary and fixed. Using (1.17), (2.1), Fubini's theorem and the change of variables: $y_{1}=2^{j_{1}} x_{1}-k_{1}$ and $y_{2}=2^{j_{2}} x_{2}-k_{2}$, one gets that

$$
\mathcal{K}_{j_{1}, j_{2}}^{k_{1}, k_{2}}(t)=2^{j_{1}\left(1-H_{1}\right)+j_{2}\left(1-H_{2}\right)} A_{j_{1}, j_{2}}^{k_{1}, k_{2}}(t),
$$

where

$$
A_{j_{1}, j_{2}}^{k_{1}, k_{2}}(t)=\int_{0}^{t} \Psi_{H_{1}}\left(2^{j_{1}} s-k_{1}\right) \Psi_{H_{2}}\left(2^{j_{2}} s-k_{2}\right) d s
$$

Let us now give, for every $\left(j_{1}, j_{2}, k_{1}, k_{2}\right) \in \mathbb{Z}^{4}$, a nice expression for the random variable $\varepsilon_{j_{1}, j_{2}}^{k_{1}, k_{2}}$ (see (1.18)). The following remark shows that it is the product of two independent standard Gaussian random variables except in the particular case where $j_{1}=j_{2}$ and $k_{1}=k_{2}$.

Remark 2.3 It is well-known (see e.g. Lemma A.1 in [19] or [14, 18]) that, for every real-valued functions $f$ and $g$ belonging to $L^{2}(\mathbb{R})$, one has

$$
\int_{\mathbb{R}^{2}}^{\prime} f\left(x_{1}\right) g\left(x_{2}\right) d B\left(x_{1}\right) d B\left(x_{2}\right)=\int_{\mathbb{R}} f(x) d B(x) \int_{\mathbb{R}} g(x) d B(x)-\int_{\mathbb{R}} f(x) g(x) d x,
$$

where $\int_{\mathbb{R}}(\cdot) d B$ denotes the usual Wiener integral on $\mathbb{R}$. Thus, using (1.18), (2.7) and the orthonormality property of the wavelets $2^{j / 2} \psi\left(2^{j} x-k\right),(j, k) \in \mathbb{Z}^{2}$, one obtains

$$
\varepsilon_{j, j}^{k, k}=\left(2^{j / 2} \int_{\mathbb{R}} \psi\left(2^{j} x-k\right) d B(x)\right)^{2}-1, \quad \text { for all }(j, k) \in \mathbb{Z}^{2},
$$

and, for every $\left(j_{1}, j_{2}, k_{1}, k_{2}\right) \in \mathbb{Z}^{4}$

$\varepsilon_{j_{1}, j_{2}}^{k_{1}, k_{2}}=\left(2^{j_{1} / 2} \int_{\mathbb{R}} \psi\left(2^{j_{1}} x-k_{1}\right) d B(x)\right)\left(2^{j_{2} / 2} \int_{\mathbb{R}} \psi\left(2^{j_{2}} x-k_{2}\right) d B(x)\right), \quad$ if $j_{1} \neq j_{2}$ or $k_{1} \neq k_{2}$.

Also, observe that it follows from the orthonormality property of the wavelets $2^{j / 2} \psi\left(2^{j} x-k\right)$, $(j, k) \in \mathbb{Z}^{2}$, and from elementary properties of the usual Wiener integral that the real-valued random variables $2^{j / 2} \int_{\mathbb{R}} \psi\left(2^{j} x-k\right) d B(x),(j, k) \in \mathbb{Z}^{2}$, are independent with the same $\mathcal{N}(0,1)$ Gaussian distribution.

The following crucial lemma easily results from Remark 2.3 and from Lemma 2 in [3]. 
Lemma 2.4 There exist $\Omega^{*}$ an event of probability 1 and $C$ a positive random variable of finite moment of any order, such that, for all $\omega \in \Omega^{*}$ and for each $\left(j_{1}, j_{2}, k_{1}, k_{2}\right) \in \mathbb{Z}^{4}$, one has

$$
\left|\varepsilon_{j_{1}, j_{2}}^{k_{1}, k_{2}}(\omega)\right| \leq C(\omega) \sqrt{\log \left(3+\left|j_{1}\right|+\left|k_{1}\right|\right) \log \left(3+\left|j_{2}\right|+\left|k_{2}\right|\right)}
$$

Let us now introduce a partition of the set of integers $\mathbb{Z}$ which will play a fundamental role in the proof of Theorem 1.2.

Definition 2.5 The parameter $a \in(1 / 2,1)$ is arbitrary and fixed once and for all. For every $(j, k) \in \mathbb{Z}_{+} \times \mathbb{Z}$, one denotes by $B_{j, k}$ the compact interval of real line centered at the dyadic number $2^{-j} k$ and of radius $2^{-j a}$, that is

$$
B_{j, k}:=\left[2^{-j} k-2^{-j a}, 2^{-j} k+2^{-j a}\right] .
$$

For each fixed $j \in \mathbb{Z}_{+}$and $t \in \mathbb{R}_{+}, D_{j}^{1}(t), D_{j}^{2}(t)$ and $D_{j}^{3}(t)$ are the three disjoint subsets of $\mathbb{Z}$ defined as:

$$
\begin{gathered}
D_{j}^{1}(t):=\left\{k \in \mathbb{Z}: B_{j, k} \subseteq[0, t]\right\}, \\
D_{j}^{2}(t):=\left\{k \in \mathbb{Z} \backslash D_{j}^{1}(t): B_{j, k} \cap[0, t] \neq \emptyset\right\},
\end{gathered}
$$

and

$$
D_{j}^{3}(t):=\left\{k \in \mathbb{Z}: B_{j, k} \cap[0, t]=\emptyset\right\} .
$$

It can easily be seen that these three disjoints sets, which depend on $t$ and also on the parameter $a$, form a partition of $\mathbb{Z}$, that is:

$$
\mathbb{Z}=D_{j}^{1}(t) \cup D_{j}^{2}(t) \cup D_{j}^{3}(t)
$$

Remark 2.6 Notice that, $D_{j}^{3}(t)$ is always an infinite set; while $D_{j}^{2}(t)$ and $D_{j}^{1}(t)$ are always finite sets which can even be empty. Their cardinalities satisfy, for each fixed positive real number $T$ and for all $j \in \mathbb{Z}_{+}$,

$$
\sup _{t \in[0, T]}\left\{\operatorname{card}\left(D_{j}^{1}(t)\right)\right\} \leq c^{\prime} 2^{j}
$$

and

$$
\sup _{t \in[0, T]}\left\{\operatorname{card}\left(D_{j}^{2}(t)\right)\right\} \leq c^{\prime \prime} 2^{j(1-a)},
$$

where $c^{\prime} \geq 1$ and $c^{\prime \prime} \geq 1$ are two finite constants. Notice that $c^{\prime}$ depends on $T$ while $c^{\prime \prime}$ does not depend on it. 
Definition 2.7 For all $J \in \mathbb{N}$, one denotes by $\aleph_{1, J}$ and $\aleph_{2, J}$ the two subsets of $\mathbb{Z}^{2}$ defined as:

$$
\aleph_{1, J}:=\left\{\left(j_{1}, j_{2}\right) \in \mathbb{Z}^{2}: j_{1} \geq j_{2} \text { and } j_{1} \geq J\right\}
$$

and

$$
\aleph_{2, J}:=\left\{\left(j_{1}, j_{2}\right) \in \mathbb{Z}^{2}: j_{2} \geq j_{1} \text { and } j_{2} \geq J\right\}
$$

Lemma 2.8 The real numbers $T>2$ and $L \geq 3 / 2$ are arbitrary and fixed. For every $J \in \mathbb{N}$, let $\mathcal{H}_{1, J}^{1}$ and $\mathcal{H}_{2, J}^{1}$ be the positive random variables defined as:

$$
\mathcal{H}_{1, J}^{1}:=\sum_{\left(j_{1}, j_{2}\right) \in \aleph_{1, J}} 2^{j_{1}\left(1-H_{1}\right)+j_{2}\left(1-H_{2}\right)} \sum_{k_{1} \in \mathbb{Z}} \sum_{\left|k_{2}\right|>2^{j_{1}+1} T} \sup _{t \in[0, T]}\left\{\left|A_{j_{1}, j_{2}}^{k_{1}, k_{2}}(t)\right|\right\}\left|\varepsilon_{j_{1}, j_{2}}^{k_{1}, k_{2}}\right|
$$

and

$$
\mathcal{H}_{2, J}^{1}:=\sum_{\left(j_{1}, j_{2}\right) \in \aleph_{2, J}} 2^{j_{1}\left(1-H_{1}\right)+j_{2}\left(1-H_{2}\right)} \sum_{\left|k_{1}\right|>2^{j_{2}+1} T} \sum_{k_{2} \in \mathbb{Z}} \sup _{t \in[0, T]}\left\{\left|A_{j_{1}, j_{2}}^{k_{1}, k_{2}}(t)\right|\right\}\left|\varepsilon_{j_{1}, j_{2}}^{k_{1}, k_{2}}\right| .
$$

Then, there exists an almost surely finite random variable $C$ such that, for all $J \in \mathbb{N}$ and $l \in\{1,2\}$, the following inequality holds on the event $\Omega^{*}$ :

$$
\mathcal{H}_{l, J}^{1} \leq C 2^{-J\left(H_{1}+H_{2}+L-3\right)} J \sqrt{\log (3+J)} .
$$

Proof of Lemma 2.8 One only shows that (2.22) is satisfied when $l=1$, the case where $l=2$ can be treated in the same way. Let $\left(j_{1}, j_{2}\right) \in \aleph_{1, J}$ be arbitrary and fixed. Using (2.6), (2.2), (2.10), (4.1), the triangle inequality, the inequality $j_{1} \geq j_{2}$, Lemma 4.2 , the fact that $y \mapsto(2+y)^{-L} \sqrt{\log (2+y)}$ is a decreasing function on $\mathbb{R}_{+}$, and (4.2) one gets that

$$
\begin{aligned}
& \sum_{k_{1} \in \mathbb{Z}} \sum_{\left|k_{2}\right|>2^{j_{1}+1} T} \sup _{t \in[0, T]}\left\{\left|A_{j_{1}, j_{2}}^{k_{1}, k_{2}}(t)\right|\right\}\left|\varepsilon_{j_{1}, j_{2}}^{k_{1}, k_{2}}\right| \\
& \leq C_{0} \sum_{k_{1} \in \mathbb{Z}} \sum_{\left|k_{2}\right|>2^{j_{1}+1_{T}} T} \int_{0}^{T} \frac{\sqrt{\log \left(3+j_{1}+\left|k_{1}\right|\right)}}{\left(3+\left|2^{j_{1}} s-k_{1}\right|\right)^{L}} \times \frac{\sqrt{\log \left(3+\left|j_{2}\right|+\left|k_{2}\right|\right)}}{\left(3+\left|2^{j_{2}} s-k_{2}\right|\right)^{L}} d s \\
& \leq C_{0} \int_{0}^{T}\left(\sum_{k_{1} \in \mathbb{Z}} \sum_{\left|k_{2}\right|>2^{j_{1}+1} T} \frac{\sqrt{\log \left(3+j_{1}+\left|k_{1}\right|\right)}}{\left(3+\left|2^{j_{1}} s-k_{1}\right|\right)^{L}} \times \frac{\sqrt{\log \left(3+\left|j_{2}\right|+\left|k_{2}\right|\right)}}{\left(3+\left|k_{2}\right|-2^{j_{2}} s\right)^{L}}\right) d s \\
& \leq C_{1} T 2^{L} \sqrt{\log \left(3+j_{1}+2^{j_{1}} T\right) \log \left(3+\left|j_{2}\right|\right)} \sum_{2^{j_{1}+1} T} \frac{\sqrt{\log \left(3+\left|k_{2}\right|\right)}}{\left(3+\left|k_{2}\right|\right)^{L}} \\
& \leq C_{1} T 2^{L+1} \sqrt{\log \left(3+j_{1}+2^{j_{1}} T\right) \log \left(3+\left|j_{2}\right|\right)} \int_{2^{j_{1}+1} T}^{+\infty} \frac{\sqrt{\log (2+y)}}{(2+y)^{L}} d y \\
& \leq C_{2} \sqrt{\log \left(3+\left|j_{2}\right|\right)} j_{1} 2^{-j_{1}(L-1)},
\end{aligned}
$$


where $C_{0}, C_{1}$ and $C_{2}$ are 3 positive random constants not depending on $\left(j_{1}, j_{2}\right)$ and $J$. Next, it follows from (2.18), (2.23), the triangle inequality, and (4.1) that

$$
\begin{aligned}
& \sum_{\left(j_{1}, j_{2}\right) \in \aleph_{1, J}} 2^{j_{1}\left(1-H_{1}\right)+j_{2}\left(1-H_{2}\right)} \sum_{k_{1} \in \mathbb{Z}} \sum_{\left|k_{2}\right|>2^{j_{1}+1} T} \sup _{t \in[0, T]}\left\{\left|A_{j_{1}, j_{2}}^{k_{1}, k_{2}}(t)\right|\right\}\left|\varepsilon_{j_{1}, j_{2}}^{k_{1}, k_{2}}\right| \\
\leq & C_{2} \sum_{j_{1}=J}^{+\infty} \sum_{j_{2}=-\infty}^{j_{1}} \sqrt{\log \left(3+\left|j_{2}\right|\right)} 2^{j_{2}\left(1-H_{2}\right)} j_{1} 2^{-j_{1}\left(H_{1}+L-2\right)} \\
\leq & C_{2} \sum_{j_{1}=J}^{+\infty} \sum_{p=0}^{+\infty} \sqrt{\log \left(3+\left|j_{1}-p\right|\right)} 2^{\left(j_{1}-p\right)\left(1-H_{2}\right)} j_{1} 2^{-j_{1}\left(H_{1}+L-2\right)} \\
\leq & C_{2} \sum_{j_{1}=J}^{+\infty} j_{1} 2^{-j_{1}\left(H_{1}+H_{2}+L-3\right)} \sum_{p=0}^{+\infty} \sqrt{\log \left(3+j_{1}+p\right)} 2^{-p\left(1-H_{2}\right)} \\
\leq & C_{3} \sum_{j_{1}=J}^{+\infty} j_{1} \sqrt{\log \left(3+j_{1}\right)} 2^{-j_{1}\left(H_{1}+H_{2}+L-3\right)} \\
\leq & C_{4} J \sqrt{\log (3+J)} 2^{-J\left(H_{1}+H_{2}+L-3\right)},
\end{aligned}
$$

where the finite random constants $C_{3}$ and $C_{4}$ are defined as:

$C_{3}:=C_{2} \sum_{p=0}^{+\infty} \sqrt{\log (3+p)} 2^{-p\left(1-H_{2}\right)} \quad$ and $\quad C_{4}:=C_{3} \sum_{q=0}^{+\infty}(1+q) \sqrt{\log (3+q)} 2^{-q\left(H_{1}+H_{2}+L-3\right)}$.

Thus, one obtains $(2.22)$ when $l=1$.

Lemma 2.9 The real numbers $T>2$ and $L \geq 2^{-1}(1-a)^{-1}+1$ are arbitrary and fixed. For every $J \in \mathbb{N}$, let $\mathcal{H}_{1, J}^{2}$ and $\mathcal{H}_{2, J}^{2}$ be the positive random variables defined as:

$$
\mathcal{H}_{1, J}^{2}:=\sum_{\left(j_{1}, j_{2}\right) \in \aleph_{1, J}} 2^{j_{1}\left(1-H_{1}\right)+j_{2}\left(1-H_{2}\right)} \sup _{t \in[0, T]}\left\{\sum_{k_{1} \in D_{j_{1}}^{3}(t)} \sum_{k_{2} \in \mathbb{Z}}\left|A_{j_{1}, j_{2}}^{k_{1}, k_{2}}(t)\right|\left|\varepsilon_{j_{1}, j_{2}}^{k_{1}, k_{2}}\right|\right\}
$$

and

$$
\mathcal{H}_{2, J}^{2}:=\sum_{\left(j_{1}, j_{2}\right) \in \aleph_{2, J}} 2^{j_{1}\left(1-H_{1}\right)+j_{2}\left(1-H_{2}\right)} \sup _{t \in[0, T]}\left\{\sum_{k_{1} \in \mathbb{Z}} \sum_{k_{2} \in D_{j_{2}}^{3}(t)}\left|A_{j_{1}, j_{2}}^{k_{1}, k_{2}}(t)\right|\left|\varepsilon_{j_{1}, j_{2}}^{k_{1}, k_{2}}\right|\right\} .
$$

Then, there exists an almost surely finite random variable $C$ such that, for all $J \in \mathbb{N}$ and $l \in\{1,2\}$, the following inequality holds on the event $\Omega^{*}$ :

$$
\mathcal{H}_{l, J}^{2} \leq C 2^{-J\left((L-1)(1-a)+H_{1}+H_{2}-2\right)} J^{2} \sqrt{\log (3+J)} .
$$


Proof of Lemma 2.9 One only shows that (2.26) is satisfied when $l=1$, the case where $l=2$ can be treated in the same way. Let $t \in[0, T]$ and $\left(j_{1}, j_{2}\right) \in \aleph_{1, J}$ be arbitrary and fixed. Using (2.6), (2.10), (2.2), Lemma 4.2 and Lemma 4.3, one gets that

$$
\begin{aligned}
& \sum_{k_{1} \in D_{j_{1}}^{3}(t)} \sum_{k_{2} \in \mathbb{Z}}\left|A_{j_{1}, j_{2}}^{k_{1}, k_{2}}(t)\right|\left|\varepsilon_{j_{1}, j_{2}}^{k_{1}, k_{2}}\right| \\
\leq & C_{0} \int_{0}^{T}\left(\sum_{k_{1} \in D_{j_{1}}^{3}(t)} \sum_{k_{2} \in \mathbb{Z}} \frac{\sqrt{\log \left(3+j_{1}+\left|k_{1}\right|\right)}}{\left(3+\left|2^{j_{1}} s-k_{1}\right|\right)^{L}} \times \frac{\sqrt{\log \left(3+\left|j_{2}\right|+\left|k_{2}\right|\right)}}{\left(3+\left|2^{j_{2}} s-k_{2}\right|\right)^{L}}\right) d s \\
\leq & C_{1} \sqrt{\log \left(3+\left|j_{2}\right|+2^{j_{2}} T\right)} \int_{0}^{T}\left(\sum_{k_{1} \in D_{j_{1}}^{3}(t)} \frac{\sqrt{\log \left(3+j_{1}+\left|k_{1}\right|\right)}}{\left(3+\left|2^{j_{1}} s-k_{1}\right|\right)^{L}}\right) d s \\
\leq & C_{2}\left(j_{1}+1\right) 2^{-j_{1}(L-1)(1-a)} \sqrt{\log \left(3+\left|j_{2}\right|+2^{j_{2}} T\right)}
\end{aligned}
$$

where $C_{0}, C_{1}$ and $C_{2}$ are 3 positive finite random constants not depending on $t,\left(j_{1}, j_{2}\right)$ and $J$. Next, it follows from (2.18), (2.27), the triangle inequality and (4.1) that

$$
\begin{aligned}
& \sum_{\left(j_{1}, j_{2}\right) \in \aleph_{1}, J} 2^{j_{1}\left(1-H_{1}\right)+j_{2}\left(1-H_{2}\right)} \sup _{t \in[0, T]}\left\{\sum_{k_{1} \in D_{j_{1}}^{3}(t)} \sum_{k_{2} \in \mathbb{Z}}\left|A_{j_{1}, j_{2}}^{k_{1}, k_{2}}(t)\right|\left|\varepsilon_{j_{1}, j_{2}}^{k_{1}, k_{2}}\right|\right\} \\
\leq & C_{2} \sum_{j_{1}=J}^{+\infty} \sum_{j_{2}=-\infty}^{j_{1}}\left(j_{1}+1\right) 2^{-j_{1}\left((L-1)(1-a)+H_{1}-1\right)} 2^{j_{2}\left(1-H_{2}\right)} \sqrt{\log \left(3+\left|j_{2}\right|+2^{j_{2}} T\right)} \\
\leq & C_{2} \sum_{j_{1}=J}^{+\infty} \sum_{p=0}^{+\infty}\left(j_{1}+1\right) 2^{-j_{1}\left((L-1)(1-a)+H_{1}-1\right)} 2^{\left(j_{1}-p\right)\left(1-H_{2}\right)} \sqrt{\log \left(3+\left|j_{1}-p\right|+2^{j_{1}-p} T\right)} \\
\leq & C_{2} \sum_{j_{1}=J}^{+\infty}\left(j_{1}+1\right) \sqrt{\log \left(3+2^{j_{1}} T\right)} 2^{-j_{1}\left((L-1)(1-a)+H_{1}+H_{2}-2\right)} \sum_{p=0}^{+\infty} \sqrt{\log \left(3+j_{1}+p\right)} 2^{-p\left(1-H_{2}\right)} \\
\leq & C_{3} \sum_{j_{1}=J}^{+\infty} j_{1}^{2} \sqrt{\log \left(3+j_{1}\right)} 2^{-j_{1}\left((L-1)(1-a)+H_{1}+H_{2}-2\right)} \\
\leq & C_{4} J^{2} \sqrt{\log (3+J)} 2^{-J\left((L-1)(1-a)+H_{1}+H_{2}-2\right)},
\end{aligned}
$$

where the finite random constants $C_{3}$ and $C_{4}$ are defined as:

$$
C_{3}:=16 C_{2}(2+T) \sum_{p=0}^{+\infty} \sqrt{\log (3+p)} 2^{-p\left(1-H_{2}\right)}
$$

and

$$
C_{4}:=C_{3} \sum_{q=0}^{+\infty}(1+q)^{2} \sqrt{\log (3+q)} 2^{-q\left((L-1)(1-a)+H_{1}+H_{2}-2\right)} .
$$

Thus one obtains (2.26) when $l=1$. 
Lemma 2.10 The real number $T>2$ is arbitrary and fixed. For every $J \in \mathbb{N}$, let $\mathcal{H}_{1, J}^{3}$ and $\mathcal{H}_{2, J}^{3}$ be the positive random variables defined as:

$$
\mathcal{H}_{1, J}^{3}:=\sum_{\left(j_{1}, j_{2}\right) \in \aleph_{1, J}} 2^{j_{1}\left(1-H_{1}\right)+j_{2}\left(1-H_{2}\right)} \sup _{t \in[0, T]}\left\{\sum_{k_{1} \in D_{j_{1}}^{2}(t)} \sum_{k_{2} \in \mathbb{Z}}\left|A_{j_{1}, j_{2}}^{k_{1}, k_{2}}(t)\right|\left|\varepsilon_{j_{1}, j_{2}}^{k_{1}, k_{2}}\right|\right\}
$$

and

$$
\mathcal{H}_{2, J}^{3}:=\sum_{\left(j_{1}, j_{2}\right) \in \aleph_{2, J}} 2^{j_{1}\left(1-H_{1}\right)+j_{2}\left(1-H_{2}\right)} \sup _{t \in[0, T]}\left\{\sum_{k_{1} \in \mathbb{Z}} \sum_{k_{2} \in D_{j_{2}}^{2}(t)}\left|A_{j_{1}, j_{2}}^{k_{1}, k_{2}}(t)\right|\left|\varepsilon_{j_{1}, j_{2}}^{k_{1}, k_{2}}\right|\right\} .
$$

Then, there exists an almost surely finite random variable $C$ such that, for all $J \in \mathbb{N}$ and $l \in\{1,2\}$, the following inequality holds on the event $\Omega^{*}$ :

$$
\mathcal{H}_{l, J}^{3} \leq C 2^{-J\left(H_{1}+H_{2}+a-2\right)} J \sqrt{\log (3+J)} .
$$

Proof of Lemma 2.10 One only shows that (2.30) is satisfied when $l=1$, the case where $l=2$ can be treated in the same way. Let $L \in(1,+\infty), t \in[0, T]$ and $\left(j_{1}, j_{2}\right) \in \aleph_{1, J}$ be arbitrary and fixed. Using (2.6), (2.10), (2.2), Lemma 4.2, the inequality $\left|k_{1}\right| \leq 2^{j_{1}(1-a)}+2^{j_{1}} T$, for all $k_{1} \in D_{j_{1}}^{2}(t)$ (see (2.13), (2.12) and (2.11)), the change of variable $z=2^{j_{1}} s-k_{1},(2.17)$ and (4.2), one gets that

$$
\begin{aligned}
& \sum_{k_{1} \in D_{j_{1}}^{2}} \sum_{k_{2} \in \mathbb{Z}}\left|A_{j_{1}, j_{2}}^{k_{1}, k_{2}}(t)\right|\left|\varepsilon_{j_{1}, j_{2}}^{k_{1}, k_{2}}\right| \\
\leq & C_{0} \int_{0}^{T}\left(\sum_{k_{1} \in D_{j_{1}}^{2}(t)} \sum_{k_{2} \in \mathbb{Z}} \frac{\sqrt{\log \left(3+j_{1}+\left|k_{1}\right|\right)}}{\left(3+\left|2^{j_{1}} s-k_{1}\right|\right)^{L}} \times \frac{\sqrt{\log \left(3+\left|j_{2}\right|+\left|k_{2}\right|\right)}}{\left(3+\left|2^{j_{2}} s-k_{2}\right|\right)^{L}}\right) d s \\
\leq & C_{1} \sqrt{\log \left(3+\left|j_{2}\right|+2^{j_{2}} T\right)} \int_{0}^{T}\left(\sum_{k_{1} \in D_{j_{1}}^{2}(t)} \frac{\sqrt{\log \left(3+j_{1}+\left|k_{1}\right|\right)}}{\left(3+\left|2^{j_{1}} s-k_{1}\right|\right)^{L}}\right) d s \\
\leq & C_{1} \sqrt{\log \left(3+\left|j_{2}\right|+2^{j_{2}} T\right) \log \left(3+j_{1}+2^{j_{1}(1-a)}+2^{j_{1}} T\right)} \sum_{k_{1} \in D_{j_{1}}^{2}(t)} \int_{\mathbb{R}\left(3+\left|2^{j_{1}} s-k_{1}\right|\right)^{L}} \\
= & C_{1}\left(\int_{\mathbb{R}} \frac{d s}{(3+|z|)^{L}}\right) \sqrt{\log \left(3+\left|j_{2}\right|+2^{j_{2}} T\right) \log \left(3+j_{1}+2^{j_{1}(1-a)}+2^{j_{1}} T\right)} \operatorname{card}\left(D_{j_{1}}^{2}(t)\right) 2^{-j_{1}} \\
\leq & C_{2} 2^{-j_{1} a} \sqrt{\left(j_{1}+1\right) \log \left(3+\left|j_{2}\right|+2^{j_{2}} T\right)},
\end{aligned}
$$

where $C_{0}, C_{1}$ and $C_{2}$ are 3 positive finite random constants not depending on $t,\left(j_{1}, j_{2}\right)$ and $J$. Next, it follows from (2.18), (2.31), (4.1) and the inequalities $a>1 / 2$ and $H_{1}+H_{2}>3 / 2$, 
that

$$
\begin{aligned}
& \sum_{\left(j_{1}, j_{2}\right) \in \aleph_{1, J}} 2^{j_{1}\left(1-H_{1}\right)+j_{2}\left(1-H_{2}\right)} \sup _{t \in[0, T]}\left\{\sum_{k_{1} \in D_{j_{1}}^{2}(t)} \sum_{k_{2} \in \mathbb{Z}}\left|A_{j_{1}, j_{2}}^{k_{1}, k_{2}}(t)\right|\left|\varepsilon_{j_{1}, j_{2}}^{k_{1}, k_{2}}\right|\right\} \\
\leq & C_{2} \sum_{j_{1}=J}^{+\infty} 2^{-j_{1}\left(H_{1}+a-1\right)} \sqrt{j_{1}+1} \sum_{j_{2}=-\infty}^{j_{1}} 2^{j_{2}\left(1-H_{2}\right)} \sqrt{\log \left(3+\left|j_{2}\right|+2^{j_{2}} T\right)} \\
\leq & C_{2} \sum_{j_{1}=J}^{+\infty} 2^{-j_{1}\left(H_{1}+a-1\right)} \sqrt{j_{1}+1} \sum_{p=0}^{+\infty} 2^{\left(j_{1}-p\right)\left(1-H_{2}\right)} \sqrt{\log \left(3+\left|j_{1}-p\right|+2^{j_{1}-p} T\right)} \\
\leq & C_{2} \sum_{j_{1}=J}^{+\infty} 2^{-j_{1}\left(H_{1}+H_{2}+a-2\right)} \sqrt{\left(j_{1}+1\right) \log \left(3+2^{j_{1}} T\right)} \sum_{p=0}^{+\infty} 2^{-p\left(1-H_{2}\right)} \sqrt{\log \left(3+j_{1}+p\right)} \\
\leq & C_{3} \sum_{j_{1}=J}^{+\infty} 2^{-j_{1}\left(H_{1}+H_{2}+a-2\right)} j_{1} \sqrt{\log \left(3+j_{1}\right)} \\
\leq & C_{4} 2^{-J\left(H_{1}+H_{2}+a-2\right)} J \sqrt{\log (3+J)}
\end{aligned}
$$

where the finite random constants $C_{3}$ and $C_{4}$ are defined as:

$$
C_{3}:=2 C_{2} \sqrt{\log (3+T)} \sum_{p=0}^{+\infty} 2^{-p\left(1-H_{2}\right)} \sqrt{\log (3+p)}
$$

and

$$
C_{4}:=C_{3} \sum_{q=0}^{+\infty} 2^{-q\left(H_{1}+H_{2}+a-2\right)}(1+q) \sqrt{\log (3+q)} .
$$

Thus, one obtains $(2.30)$ when $l=1$.

Definition 2.11 For all $\left(j_{1}, j_{2}, k_{1}, k_{2}\right) \in \mathbb{Z}^{4}$, one sets

$$
F_{j_{1}, j_{2}}^{k_{1}, k_{2}}:=\int_{\mathbb{R}} \Psi_{H_{1}}\left(2^{j_{1}} s-k_{1}\right) \Psi_{H_{2}}\left(2^{j_{2}} s-k_{2}\right) d s .
$$

One knows from Remark 2.1 that the real-valued functions $\Psi_{H_{1}}$ and $\Psi_{H_{2}}$ belong to the Schwartz class $S(\mathbb{R})$. Therefore, $F_{j_{1}, j_{2}}^{k_{1}, k_{2}}$ is well-defined and it results from the Plancherel formula and from elementary property of Fourier transform that

$$
F_{j_{1}, j_{2}}^{k_{1}, k_{2}}=2^{-j_{1}-j_{2}} \int_{\mathbb{R}} \exp \left(-i\left(2^{-j_{1}} k_{1}-2^{-j_{2}} k_{2}\right) \xi\right) \widehat{\Psi}_{H_{1}}\left(2^{-j_{1}} \xi\right) \overline{\widehat{\Psi}_{H_{2}}\left(2^{-j_{2}} \xi\right)} d \xi .
$$


Remark 2.12 Observe that one can easily derive from (2.4) and (2.33) that

$$
F_{j_{1}, j_{2}}^{k_{1}, k_{2}}=0, \quad \text { for all }\left(j_{1}, j_{2}, k_{1}, k_{2}\right) \in \mathbb{Z}^{4} \text { such that }\left|j_{1}-j_{2}\right| \geq 2 .
$$

In other words, a necessary condition for having $F_{j_{1}, j_{2}}^{k_{1}, k_{2}} \neq 0$ is that $j_{2} \in\left\{j_{1}-1, j_{1}, j_{1}+1\right\}$. This leads us to introduce the following notations: for every $\left(j, k_{1}, k_{2}\right) \in \mathbb{Z}^{3}$, one sets

$$
\tilde{F}_{j}^{k_{1}, k_{2}}:=F_{j, j}^{k_{1}, k_{2}}, \quad \grave{F}_{j}^{k_{1}, k_{2}}:=F_{j, j-1}^{k_{1}, k_{2}} \quad \text { and } \quad \dot{F}_{j}^{k_{1}, k_{2}}:=F_{j, j+1}^{k_{1}, k_{2}} .
$$

Thus, using (2.33) and the change of variables $\eta=2^{-j} \xi$ and $\eta^{\prime}=2^{-j-1} \xi$, one gets that

$$
\begin{aligned}
& \tilde{F}_{j}^{k_{1}, k_{2}}:=F_{j, j}^{k_{1}, k_{2}}=2^{-j} \int_{\mathbb{R}} \exp \left(-i\left(k_{1}-k_{2}\right) \eta\right) \widehat{\Psi}_{H_{1}}(\eta) \overline{\widehat{\Psi}_{H_{2}}(\eta)} d \eta, \\
& \grave{F}_{j}^{k_{1}, k_{2}}:=F_{j, j-1}^{k_{1}, k_{2}}=2^{1-j} \int_{\mathbb{R}} \exp \left(-i\left(k_{1}-2 k_{2}\right) \eta\right) \widehat{\Psi}_{H_{1}}(\eta) \widehat{\Psi}_{H_{2}}(2 \eta) d \eta
\end{aligned}
$$

and

$$
\dot{F}_{j}^{k_{1}, k_{2}}:=F_{j, j+1}^{k_{1}, k_{2}}=2^{-j} \int_{\mathbb{R}} \exp \left(-i\left(2 k_{1}-k_{2}\right) \eta^{\prime}\right) \widehat{\Psi}_{H_{1}}\left(2 \eta^{\prime}\right) \overline{\widehat{\Psi}_{H_{2}}\left(\eta^{\prime}\right)} d \eta^{\prime} .
$$

Remark 2.13 Let $\Lambda_{1}, \Lambda_{2}$ and $\Lambda_{3}$ be the three functions defined, for all $\eta \in \mathbb{R}$, as:

$$
\Lambda_{1}(\eta):=\widehat{\Psi}_{H_{1}}(\eta) \overline{\widehat{\Psi}_{H_{2}}(\eta)}, \quad \Lambda_{2}(\eta):=\widehat{\Psi}_{H_{1}}(\eta) \overline{\widehat{\Psi}_{H_{2}}(2 \eta)} \quad \text { and } \quad \Lambda_{3}(\eta):=\widehat{\Psi}_{H_{1}}(2 \eta) \overline{\widehat{\Psi}_{H_{2}}(\eta)} .
$$

These three functions as well as their Fourier transforms $\widehat{\Lambda}_{1}, \widehat{\Lambda}_{2}$ and $\widehat{\Lambda}_{3}$ belong to the Schwartz class $S(\mathbb{R})$ since $\Psi_{H_{1}}$ and $\Psi_{H_{2}}$ are in this class. Thus, for any arbitrary fixed positive real number $L$, there exists a positive constant $c$ such that

$$
\left|\widehat{\Lambda}_{m}(v)\right| \leq c(3+|v|)^{-L}, \quad \text { for all }(m, v) \in\{1,2,3\} \times \mathbb{R} .
$$

On the other hand, one knows from $(2.35),(2.36),(2.37)$ and $(2.38)$, that, for all $\left(j, k_{1}, k_{2}\right) \in$ $\mathbb{Z}^{3}$, one has

$$
\tilde{F}_{j}^{k_{1}, k_{2}}=2^{-j} \widehat{\Lambda}_{1}\left(k_{1}-k_{2}\right), \quad \grave{F}_{j}^{k_{1}, k_{2}}=2^{1-j} \widehat{\Lambda}_{1}\left(k_{1}-2 k_{2}\right) \quad \text { and } \quad \dot{F}_{j}^{k_{1}, k_{2}}=2^{-j} \widehat{\Lambda}_{1}\left(2 k_{1}-k_{2}\right) .
$$

Thus, one can derive from (2.39) and (2.40) that, for all $\left(j, k_{1}, k_{2}\right) \in \mathbb{Z}^{3}$, the following three inequalities, in which $c$ denotes the same constant as in (2.39), hold:

$$
\begin{gathered}
\left|\tilde{F}_{j}^{k_{1}, k_{2}}\right| \leq c 2^{-j}\left(3+\left|k_{1}-k_{2}\right|\right)^{-L} \\
\left|\grave{F}_{j}^{k_{1}, k_{2}}\right| \leq c 2^{1-j}\left(3+\left|k_{1}-2 k_{2}\right|\right)^{-L} \text { and }\left|\dot{F}_{j}^{k_{1}, k_{2}}\right| \leq c 2^{-j}\left(3+\left|2 k_{1}-k_{2}\right|\right)^{-L} .
\end{gathered}
$$


Lemma 2.14 The real numbers $L>2$ and $T>2$ are arbitrary and fixed. For every $J \in \mathbb{N}$, let $\mathcal{H}_{1, J}^{4}$ and $\mathcal{H}_{2, J}^{4}$ be the positive random variables defined as:

$$
\mathcal{H}_{1, J}^{4}:=\sum_{\left(j_{1}, j_{2}\right) \in \aleph_{1, J}} 2^{j_{1}\left(1-H_{1}\right)+j_{2}\left(1-H_{2}\right)} \sup _{t \in[0, T]}\left\{\sum_{k_{1} \in D_{j_{1}}^{1}(t)} \sum_{k_{2} \in \mathbb{Z}}\left|A_{j_{1}, j_{2}}^{k_{1}, k_{2}}(t)-F_{j_{1}, j_{2}}^{k_{1}, k_{2}}\right|\left|\varepsilon_{j_{1}, j_{2}}^{k_{1}, k_{2}}\right|\right\}
$$

and

$$
\mathcal{H}_{2, J}^{4}:=\sum_{\left(j_{1}, j_{2}\right) \in \aleph_{2, J}} 2^{j_{1}\left(1-H_{1}\right)+j_{2}\left(1-H_{2}\right)} \sup _{t \in[0, T]}\left\{\sum_{k_{1} \in \mathbb{Z}} \sum_{k_{2} \in D_{j_{2}}^{1}(t)}\left|A_{j_{1}, j_{2}}^{k_{1}, k_{2}}(t)-F_{j_{1}, j_{2}}^{k_{1}, k_{2}}\right|\left|\varepsilon_{j_{1}, j_{2}}^{k_{1}, k_{2}}\right|\right\} .
$$

Then, there exists an almost surely finite random variable $C$ such that, for all $J \in \mathbb{N}$ and $l \in\{1,2\}$, the following inequality holds on the event $\Omega^{*}$ :

$$
\mathcal{H}_{l, J}^{4} \leq C J^{3 / 2} 2^{-J\left((L-2)(1-a)+H_{1}+H_{2}-1\right)} .
$$

Proof of Lemma 2.14 One only shows that (2.45) is satisfied when $l=1$, the case where $l=2$ can be treated in the same way. Let $t \in[0, T]$ and $\left(j_{1}, j_{2}\right) \in \aleph_{1, J}$ be arbitrary and fixed. Using (2.6), (2.32), (2.11), (2.12), (2.2), (2.10), the inequality $\left|k_{1}\right| \leq 2^{j_{1}} T$ when $k_{1} \in D_{j_{1}}^{1}(t)$, the inequality $2^{j_{1}} T \geq j_{1}$, Lemma 4.2 , the inequality $j_{2} \leq j_{1}$ and (4.1), one gets that

$$
\begin{aligned}
& \sum_{k_{1} \in D_{j_{1}}^{1}(t)} \sum_{k_{2} \in \mathbb{Z}}\left|A_{j_{1}, j_{2}}^{k_{1}, k_{2}}(t)-F_{j_{1}, j_{2}}^{k_{1}, k_{2}}\right|\left|\varepsilon_{j_{1}, j_{2}}^{k_{1}, k_{2}}\right| \\
\leq & C_{0} \sqrt{\log \left(3+2^{j_{1}+1} T\right)} \int_{\mathbb{R} \backslash[0, t]}\left(\sum_{k_{1} \in D_{j_{1}}^{1}(t)} \frac{1}{\left(3+\left|2^{j_{1}} s-k_{1}\right|\right)^{L}}\right)\left(\sum_{k_{2} \in \mathbb{Z}} \frac{\sqrt{\log \left(3+\left|j_{2}\right|+\left|k_{2}\right|\right)}}{\left(3+\left|2^{j_{2}} s-k_{2}\right|\right)^{L}}\right) d s \\
\leq & C_{1} \sqrt{\log \left(3+2^{j_{1}+1} T\right)} \int_{\mathbb{R} \backslash[0, t]}\left(\sum_{k_{1} \in D_{j_{1}}^{1}(t)} \frac{\sqrt{\log \left(3+\left|j_{2}\right|+\left|2^{j_{2}} s\right|\right)}}{\left(3+\left|2^{j_{1}} s-k_{1}\right|\right)^{L}}\right) d s \\
\leq & C_{1} \sqrt{\log \left(3+2^{j_{1}+1} T\right)} \int_{\mathbb{R} \backslash[0, t]}\left(\sum_{k_{1} \in D_{j_{1}}^{1}(t)} \frac{\sqrt{\log \left(3+\left|j_{2}\right|+\left|2^{j_{1}} s\right|\right)}}{\left(3+\left|2^{j_{1}} s-k_{1}\right|\right)^{L}}\right) d s \\
\leq & C_{1} \log \left(3+2^{j_{1}+1} T\right) \int_{\mathbb{R} \backslash[0, t]}\left(\sum_{k_{1} \in D_{j_{1}}^{1}(t)} \frac{\sqrt{\log \left(3+\left|j_{2}\right|+\left|2^{j_{1}} s-k_{1}\right|\right)}}{\left(3+\left|2^{j_{1}} s-k_{1}\right|\right)^{L}}\right) d s \\
\leq & C_{1} \log \left(3+2^{j_{1}+1} T\right)\left(U_{j_{1}, j_{2}}(t)+V_{j_{1}, j_{2}}\right),
\end{aligned}
$$

where $C_{0}$ and $C_{1}$ are 2 positive finite random constants not depending on $t, T,\left(j_{1}, j_{2}\right)$ and $J$, and where

$$
U_{j_{1}, j_{2}}(t):=\int_{t}^{+\infty}\left(\sum_{k_{1} \leq 2^{j_{1}} t-2^{j_{1}(1-a)}} \frac{\sqrt{\log \left(3+\left|j_{2}\right|+2^{j_{1}} s-k_{1}\right)}}{\left(3+2^{j_{1}} s-k_{1}\right)^{L}}\right) d s
$$


and

$$
V_{j_{1}, j_{2}}:=\int_{-\infty}^{0}\left(\sum_{k_{1} \geq 2^{j_{1}(1-a)}} \frac{\sqrt{\log \left(3+\left|j_{2}\right|-2^{j_{1}} s+k_{1}\right)}}{\left(3-2^{j_{1}} s+k_{1}\right)^{L}}\right) d s .
$$

Next, the change of variable $y=2^{j_{1}}(s-t)$ in $(2.47)$, the fact that, for any fixed $j_{2} \in \mathbb{Z}$, the function $y \mapsto(2+y)^{-L} \sqrt{\log \left(2+\left|j_{2}\right|+y\right)}$ is decreasing on $\mathbb{R}_{+}$, and integrations by parts entail that

$$
\begin{aligned}
& U_{j_{1}, j_{2}}(t)=2^{-j_{1}} \int_{0}^{+\infty}\left(\sum_{k_{1} \leq 2^{j_{1}} t-2^{j_{1}(1-a)}} \frac{\sqrt{\log \left(3+\left|j_{2}\right|+y+2^{j_{1}} t-k_{1}\right)}}{\left(3+y+2^{j_{1}} t-k_{1}\right)^{L}}\right) d y \\
& \leq 2^{-j_{1}} \int_{0}^{+\infty}\left(\sum_{m=0}^{+\infty} \frac{\sqrt{\log \left(3+\left|j_{2}\right|+y+2^{j_{1}(1-a)}+m\right)}}{\left(3+y+2^{j_{1}(1-a)}+m\right)^{L}}\right) d y \\
& \leq 2^{-j_{1}} \int_{0}^{+\infty}\left(\int_{0}^{+\infty} \frac{\sqrt{\log \left(2+\left|j_{2}\right|+y+2^{j_{1}(1-a)}+z\right)}}{\left(2+y+2^{j_{1}(1-a)}+z\right)^{L}} d z\right) d y \\
& \leq 2^{1-j_{1}} \int_{0}^{+\infty} \frac{\sqrt{\log \left(2+\left|j_{2}\right|+y+2^{j_{1}(1-a)}\right)}}{\left(2+y+2^{j_{1}(1-a)}\right)^{L-1}} d y \\
& \leq 2^{2-j_{1}}(L-2)^{-1} \frac{\sqrt{\log \left(2+\left|j_{2}\right|+2^{j_{1}(1-a)}\right)}}{\left(2+2^{j_{1}(1-a)}\right)^{L-2}}
\end{aligned}
$$

Similar arguments allow to derive from (2.48) that

$$
V_{j_{1}, j_{2}} \leq 2^{2-j_{1}}(L-2)^{-1} \frac{\sqrt{\log \left(2+\left|j_{2}\right|+2^{j_{1}(1-a)}\right)}}{\left(2+2^{j_{1}(1-a)}\right)^{L-2}} .
$$

Next, it follows from (2.18), (2.46), (2.49), (2.50), the triangle inequality, (4.1) and (1.11) that

$$
\begin{aligned}
& \sum_{\left(j_{1}, j_{2}\right) \in \aleph_{1, J}} 2^{j_{1}\left(1-H_{1}\right)+j_{2}\left(1-H_{2}\right)} \sup _{t \in[0, T]}\left\{\sum_{k_{1} \in D_{j_{1}}^{1}(t)} \sum_{k_{2} \in \mathbb{Z}}\left|A_{j_{1}, j_{2}}^{k_{1}, k_{2}}(t)-F_{j_{1}, j_{2}}^{k_{1}, k_{2}}\right|\left|\varepsilon_{j_{1}, j_{2}}^{k_{1}, k_{2}}\right|\right\} \\
\leq & C_{2} \sum_{j_{1}=J}^{+\infty} \sum_{j_{2}=-\infty}^{j_{1}}\left(j_{1}+1\right) 2^{-j_{1}\left((L-2)(1-a)+H_{1}\right)} 2^{j_{2}\left(1-H_{2}\right)} \sqrt{\log \left(2+\left|j_{2}\right|+2^{j_{1}(1-a)}\right)} \\
\leq & C_{2} \sum_{j_{1}=J}^{+\infty} \sum_{p=0}^{+\infty}\left(j_{1}+1\right) 2^{-j_{1}\left((L-2)(1-a)+H_{1}\right)} 2^{\left(j_{1}-p\right)\left(1-H_{2}\right)} \sqrt{\log \left(2+\left|j_{1}-p\right|+2^{j_{1}(1-a)}\right)} \\
\leq & C_{2} \sum_{j_{1}=J}^{+\infty}\left(j_{1}+1\right) 2^{-j_{1}\left((L-2)(1-a)+H_{1}+H_{2}-1\right)} \sum_{p=0}^{+\infty} 2^{-p\left(1-H_{2}\right)} \sqrt{\log \left(2+j_{1}+2^{j_{1}(1-a)}+p\right)} \\
\leq & C_{3} \sum_{j_{1}=J}^{+\infty} j_{1}^{3 / 2} 2^{-j_{1}\left((L-2)(1-a)+H_{1}+H_{2}-1\right)} \\
\leq & C_{4} J^{3 / 2} 2^{-J\left((L-2)(1-a)+H_{1}+H_{2}-1\right)},
\end{aligned}
$$


where $C_{2}, C_{3}$ and $C_{4}$ are 3 positive finite random constants not depending on $t$ and $J$. Thus one obtains $(2.45)$ when $l=1$.

Definition 2.15 For all $J \in \mathbb{N}$, one denotes by $\mathcal{M}_{1, J}$ and $\mathcal{M}_{2, J}$ the positive random variables defined as:

$$
\mathcal{M}_{1, J}:=\sum_{\left(j_{1}, j_{2}\right) \in \aleph_{1, J}} 2^{j_{1}\left(1-H_{1}\right)+j_{2}\left(1-H_{2}\right)} \sup _{t \in[0, T]}\left\{\left|\sum_{k_{1} \in D_{j_{1}}^{1}(t)} \sum_{\left|k_{2}\right| \leq 2^{j_{1}+1} T} F_{j_{1}, j_{2}}^{k_{1}, k_{2}} \varepsilon_{j_{1}, j_{2}}^{k_{1}, k_{2}}\right|\right\}
$$

and

$$
\mathcal{M}_{2, J}:=\sum_{\left(j_{1}, j_{2}\right) \in \aleph_{2, J}} 2^{j_{1}\left(1-H_{1}\right)+j_{2}\left(1-H_{2}\right)} \sup _{t \in[0, T]}\left\{\left|\sum_{\left|k_{1}\right| \leq 2^{j_{2}+1} T} \sum_{k_{2} \in D_{j_{2}}^{1}(t)} F_{j_{1}, j_{2}}^{k_{1}, k_{2}} \varepsilon_{j_{1}, j_{2}}^{k_{1}, k_{2}}\right|\right\},
$$

where the real number $T>2$ is arbitrary and fixed.

Lemma 2.16 There exists $\Omega^{* *}$ an event of probability 1 , and there is $C^{* *}$ a positive finite random variable, such that, for all $(\omega, J) \in \Omega^{* *} \times \mathbb{N}$ and $l \in\{1,2\}$, one has

$$
\mathcal{M}_{l, J}(\omega) \leq C^{* *}(\omega) J 2^{-J\left(H_{1}+H_{2}-3 / 2\right)} .
$$

Our next goal is to show that Lemma 2.16 is satisfied; one focuses on the case $l=1$, the other case $l=2$ can be treated similarly.

Remark 2.17 Observe that setting $j=j_{1}$ in (2.51), and using Remark 2.12 and (2.18) one has that

$$
\mathcal{M}_{1, J}=\sum_{j=J}^{+\infty} 2^{j\left(2-H_{1}-H_{2}\right)}\left(\sup _{t \in[0, T]}\left\{\tilde{M}_{j}(t)\right\}+2^{H_{2}-1} \sup _{t \in[0, T]}\left\{\grave{M}_{j}(t)\right\}\right),
$$

where, for all $j \in \mathbb{N}$ and $t \in[0, T]$,

$$
\tilde{M}_{j}(t):=\sum_{k_{1} \in D_{j}^{1}(t)} \sum_{\left|k_{2}\right| \leq 2^{j+1} T} \tilde{F}_{j}^{k_{1}, k_{2}} \varepsilon_{j, j}^{k_{1}, k_{2}}
$$

and

$$
\grave{M}_{j}(t):=\sum_{k_{1} \in D_{j}^{1}(t)} \sum_{\left|k_{2}\right| \leq 2^{j+1} T} \grave{F}_{j}^{k_{1}, k_{2}} \varepsilon_{j, j-1}^{k_{1}, k_{2}} .
$$


In fact, in the case where $l=1$, Lemma 2.16 is a straightforward consequence of (2.54) and the following lemma.

Lemma 2.18 There exist $\tilde{\Omega}^{* *}$ and $\grave{\Omega}^{* *}$ two events of probability 1 , and there are $\tilde{C}^{* *}$ and $\grave{C}^{* *}$ two positive finite random variables, such that one has:

$$
\sup _{t \in[0, T]}\left\{\left|\tilde{M}_{j}(t, \omega)\right|\right\} \leq \tilde{C}^{* *}(\omega) j 2^{-j / 2}, \quad \text { for all }(\omega, j) \in \tilde{\Omega}^{* *} \times \mathbb{N}
$$

and

$$
\sup _{t \in[0, T]}\left\{\left|\grave{M}_{j}(t, \omega)\right|\right\} \leq \grave{C}^{* *}(\omega) j 2^{-j / 2}, \quad \text { for all }(\omega, j) \in \grave{\Omega}^{* *} \times \mathbb{N} \text {. }
$$

In order to show that Lemma 2.18 is satisfied, one needs some preliminary results.

Remark 2.19 Let $j \in \mathbb{Z}_{+}$and $t \in \mathbb{R}_{+}$be arbitrary and fixed. Using (2.11) and (2.12) it can easily be shown that

$$
D_{j}^{1}(t)=\left\{\begin{array}{l}
\emptyset, \quad \text { if } t \in\left[0,2^{1-j a}\right), \\
D_{j}^{1}\left(m_{j, t} 2^{-j}+2^{-j a}\right), \quad \text { if } t \in\left[2^{1-j a},+\infty\right),
\end{array}\right.
$$

where $m_{j, t}$ is the positive integer defined as $m_{j, t}:=\left\lfloor 2^{j} t-2^{j(1-a)}\right\rfloor$. Thus, denoting by $\mathcal{I}_{j}$ the finite set of positive integers defined as

$$
\mathcal{I}_{j}:=\mathbb{N} \cap\left(2^{j(1-a)}-1,2^{j} T-2^{j(1-a)}\right],
$$

in view of (2.59), one has, for all $j \in \mathbb{N}$, that

$$
\sup _{t \in[0, T]}\left\{\left|\tilde{M}_{j}(t)\right|\right\}=\sup _{m \in \mathcal{I}_{j}}\left\{\left|\tilde{M}_{j}\left(m 2^{-j}+2^{-j a}\right)\right|\right\}
$$

and

$$
\sup _{t \in[0, T]}\left\{\left|\grave{M}_{j}(t)\right|\right\}=\sup _{m \in \mathcal{I}_{j}}\left\{\left|\grave{M}_{j}\left(m 2^{-j}+2^{-j a}\right)\right|\right\} .
$$

Observe that the cardinality of the set $\mathcal{I}_{j}$, satisfies, for some finite constant $c \geq 1$ only depending on $T$,

$$
\operatorname{card}\left(\mathcal{I}_{j}\right) \leq c 2^{j}, \quad \text { for all } j \in \mathbb{N} \text {. }
$$


The following lemma is a straightforward consequence of Theorem 6.7 in [14].

Lemma 2.20 There exists a positive finite universal constant $\breve{c}$ such that, for every random variable $\chi$ belonging to the second order Wiener chaos and for each real number $y \geq 2$, one has

$$
\mathbb{P}\left(|\chi|>y\|\chi\|_{L^{2}(\Omega)}\right) \leq \exp (-\breve{c} y)
$$

where $\|\chi\|_{L^{2}(\Omega)}:=\left(\mathbb{E}\left[|\chi|^{2}\right]\right)^{1 / 2}$.

Lemma 2.21 There exists a finite constant $c>0$ which depends on $T$, such that one has

$$
\sup _{t \in[0, T]}\left\{\left\|\tilde{M}_{j}(t)\right\|_{L^{2}(\Omega)}\right\} \leq c 2^{-j / 2}, \quad \text { for all } j \in \mathbb{N}
$$

and

$$
\sup _{t \in[0, T]}\left\{\left\|\grave{M}_{j}(t)\right\|_{L^{2}(\Omega)}\right\} \leq c 2^{-j / 2}, \quad \text { for all } j \in \mathbb{N}
$$

Proof of Lemma 2.21 We only give the proof of (2.65) since (2.66) can be shown in the same way except that the first inequality in (2.42) has to be used instead of (2.41). Let $t \in[0, T]$ and $j \in \mathbb{N}$ be arbitrary and fixed. Observe that, in view of the inclusion $D_{j}^{1}(t) \subset\left\{k_{2} \in \mathbb{Z}:\left|k_{2}\right| \leq 2^{j+1} T\right\}$ the set $D_{j}^{1}(t) \times\left\{k_{2} \in \mathbb{Z}:\left|k_{2}\right| \leq 2^{j+1} T\right\}$ can be expressed as the disjoint union:

$$
D_{j}^{1}(t) \times\left\{k_{2} \in \mathbb{Z}:\left|k_{2}\right| \leq 2^{j+1} T\right\}=\Delta_{j}^{<}(t) \cup \Delta_{j}^{=}(t) \cup \Delta_{j}^{>}(t) \cup \Delta_{j}^{c}(t),
$$

where, for any $\mathcal{R} \in\{<,=,>\}$

$$
\Delta_{j}^{\mathcal{R}}(t):=\left\{\left(k_{1}, k_{2}\right) \in D_{j}^{1}(t) \times D_{j}^{1}(t): \quad k_{1} \mathcal{R} k_{2}\right\}
$$

and

$$
\Delta_{j}^{c}(t):=D_{j}^{1}(t) \times\left\{k_{2} \in \mathbb{Z}:\left|k_{2}\right| \leq 2^{j+1} T \text { and } k_{2} \notin D_{j}^{1}(t)\right\} .
$$

Thus, using (2.55), one gets that

$$
\tilde{M}_{j}(t)=\tilde{M}_{j}^{<}(t)+\tilde{M}_{j}^{=}(t)+\tilde{M}_{j}^{>}(t)+\tilde{M}_{j}^{c}(t),
$$

where, for any $\mathcal{R} \in\{<,=,>, c\}$,

$$
\tilde{M}_{j}^{\mathcal{R}}(t):=\sum_{\left(k_{1}, k_{2}\right) \in \Delta_{j}^{\mathcal{R}}(t)} \tilde{F}_{j}^{k_{1}, k_{2}} \varepsilon_{j, j}^{k_{1}, k_{2}} .
$$


Next, it follows from (2.69) and the triangle inequality that

$$
\left\|\tilde{M}_{j}(t)\right\|_{L^{2}(\Omega)} \leq\left\|\tilde{M}_{j}^{<}(t)\right\|_{L^{2}(\Omega)}+\left\|\tilde{M}_{j}^{=}(t)\right\|_{L^{2}(\Omega)}+\left\|\tilde{M}_{j}^{>}(t)\right\|_{L^{2}(\Omega)}+\left\|\tilde{M}_{j}^{c}(t)\right\|_{L^{2}(\Omega)} .
$$

The advantage in using the sets $\Delta_{j}^{\mathcal{R}}(t), \mathcal{R} \in\{<,=,>, c\}$, is that one can derive from (2.67), (2.68) and Remark 2.3, that, for any $\left(k_{1}^{\prime}, k_{2}^{\prime}\right) \in \Delta_{j}^{\mathcal{R}}(t)$ and $\left(k_{1}^{\prime \prime}, k_{2}^{\prime \prime}\right) \in \Delta_{j}^{\mathcal{R}}(t)$, the two centered random variables $\varepsilon_{j, j}^{k_{1}^{\prime}, k_{2}^{\prime}}$ and $\varepsilon_{j, j}^{k_{1}^{\prime \prime}, k_{2}^{\prime \prime}}$ are uncorrelated except when $\left(k_{1}^{\prime}, k_{2}^{\prime}\right)=\left(k_{1}^{\prime \prime}, k_{2}^{\prime \prime}\right)$. Then, it follows from $(2.70),(2.8),(2.9)$, classical properties of Wiener integral, the fact that the fourth moment of a standard Gaussian random variable equals 3, and (2.41) that

$$
\begin{aligned}
\left\|\tilde{M}_{j}^{\mathcal{R}}(t)\right\|_{L^{2}(\Omega)}^{2} & :=\mathbb{E}\left(\left(\tilde{M}_{j}^{\mathcal{R}}(t)\right)^{2}\right) \\
& =\sum_{\left(k_{1}, k_{2}\right) \in \Delta_{j}^{\mathcal{R}}(t)}\left(\tilde{F}_{j}^{k_{1}, k_{2}}\right)^{2} \mathbb{E}\left(\left(\varepsilon_{j, j}^{k_{1}, k_{2}}\right)^{2}\right) \leq c_{1} 2^{-2 j} \sum_{\left(k_{1}, k_{2}\right) \in \Delta_{j}^{\mathcal{R}}(t)}\left(3+\left|k_{1}-k_{2}\right|\right)^{-2 L},
\end{aligned}
$$

where the arbitrary real number $L>1 / 2$ is fixed, and $c_{1}>0$ is a constant only depending on $L$. Then, denoting by $c_{2}$ the finite constant $c_{2}:=2 c_{1} \sum_{n=0}^{+\infty}(3+n)^{-2 L}$ and using (2.72) and the inclusion

$$
\Delta_{j}^{\mathcal{R}}(t) \subset \mathbb{Z} \times\left\{k_{2} \in \mathbb{Z}:\left|k_{2}\right| \leq 2^{j+1} T\right\},
$$

one obtains that

$$
\left\|\tilde{M}_{j}^{\mathcal{R}}(t)\right\|_{L^{2}(\Omega)}^{2} \leq c_{1} 2^{-2 j} \sum_{\left|k_{2}\right| \leq 2^{j+1} T} \sum_{k_{1} \in \mathbb{Z}}\left(3+\left|k_{1}-k_{2}\right|\right)^{-2 L} \leq 8 T c_{2} 2^{-j} .
$$

Finally, one can derive from (2.73) and (2.71) that (2.65) holds.

We are now in position to prove Lemma 2.18.

Proof of Lemma 2.18 We only give the proof of (2.57) since (2.58) can be shown in the same way. Let $\kappa \geq 2$ be a constant which will be defined more precisely later. Using (2.61) and (2.60), for all $j \in \mathbb{N}$, one has

$$
\begin{aligned}
& \mathbb{P}\left(\sup _{t \in[0, T]}\left\{\left|\tilde{M}_{j}(t)\right|\right\}>\kappa j \sup _{t \in[0, T]}\left\{\left\|\tilde{M}_{j}(t)\right\|_{L^{2}(\Omega)}\right\}\right) \\
& =\mathbb{P}\left(\sup _{m \in \mathcal{I}_{j}}\left\{\left|\tilde{M}_{j}\left(m 2^{-j}+2^{-j a}\right)\right|\right\}>\kappa j \sup _{t \in[0, T]}\left\{\left\|\tilde{M}_{j}(t)\right\|_{L^{2}(\Omega)}\right\}\right) \\
& \leq \sum_{m \in \mathcal{I}_{j}} \mathbb{P}\left(\left|\tilde{M}_{j}\left(m 2^{-j}+2^{-j a}\right)\right|>\kappa j \sup _{t \in[0, T]}\left\{\left\|\tilde{M}_{j}(t)\right\|_{L^{2}(\Omega)}\right\}\right) \\
& \leq \sum_{m \in \mathcal{I}_{j}} \mathbb{P}\left(\left|\tilde{M}_{j}\left(m 2^{-j}+2^{-j a}\right)\right|>\kappa j\left\|\tilde{M}_{j}\left(m 2^{-j}+2^{-j a}\right)\right\|_{L^{2}(\Omega)}\right) .
\end{aligned}
$$


Next, observe that, for any $t \in[0, T]$ (and in particular for $t=m 2^{-j}+2^{-j a}$ ) the random variable $\tilde{M}_{j}(t)$ belongs to the second order Wiener chaos, since it is (see (2.55)) a linear combination of random variables $\varepsilon_{j, j}^{k_{1}, k_{2}}$ which are in this chaos. Thus, one can make use of Lemma 2.20 in order to bound from above the probabilities in (2.74). By this way, for all $m \in \mathcal{I}_{j}$, one gets

$$
\mathbb{P}\left(\left|\tilde{M}_{j}\left(m 2^{-j}+2^{-j a}\right)\right|>\kappa j\left\|\tilde{M}_{j}\left(m 2^{-j}+2^{-j a}\right)\right\|_{L^{2}(\Omega)}\right) \leq \exp (-\breve{c} \kappa j),
$$

where $\breve{c}$ is the same positive finite constant as in (2.64). Next combining (2.74) and (2.75) with (2.63), one obtains that

$$
\begin{aligned}
& \mathbb{P}\left(\sup _{t \in[0, T]}\left\{\left|\tilde{M}_{j}(t)\right|\right\}>\kappa j \sup _{t \in[0, T]}\left\{\left\|\tilde{M}_{j}(t)\right\|_{L^{2}(\Omega)}\right\}\right) \\
& \leq c_{1} 2^{j} \exp (-\breve{c} \kappa j)=c_{1} \exp (-(\breve{c} \kappa-\log 2) j),
\end{aligned}
$$

where $c_{1}$ denotes the positive finite constant $c$ in (2.63). One can assume that the finite constant $\kappa$ is chosen such that $\kappa>(\log 2) / \breve{c}$. Then, one can derive from (2.76) that

$$
\sum_{j=1}^{+\infty} \mathbb{P}\left(\sup _{t \in[0, T]}\left\{\left|\tilde{M}_{j}(t)\right|\right\}>\kappa j \sup _{t \in[0, T]}\left\{\left\|\tilde{M}_{j}(t)\right\|_{L^{2}(\Omega)}\right\}\right)<+\infty .
$$

Thus, the Borel-Cantelli lemma implies that there exist $\tilde{\Omega}^{* *}$ an event of probability 1 and $\tilde{C}_{2}^{* *}$ a positive finite random variable, such that, for all $\omega \in \Omega^{* *}$ and for every $j \in \mathbb{N}$, one has

$$
\sup _{t \in[0, T]}\left\{\left|\tilde{M}_{j}(t, \omega)\right|\right\} \leq \tilde{C}_{2}^{* *}(\omega) j \sup _{t \in[0, T]}\left\{\left\|\tilde{M}_{j}(t)\right\|_{L^{2}(\Omega)}\right\}
$$

Finally, (2.57) results from (2.65) and (2.77).

We are now in position to complete the prove Theorem 1.2.

End of the proof of Theorem 1.2 Recall that the compact interval $I$ is assumed to be of the form $I=[0, T]$, where the real number $T>2$ is arbitrary. Also recall that $\Omega^{*}$ denotes the event of probability 1 which was introduced in Lemma 2.4. Let us first show that, for all fixed $\left(j_{1}, j_{2}\right) \in \mathbb{Z}^{2}$ and for every $\omega \in \Omega^{*}$, the series of continuous of functions $\sum_{\left(k_{1}, k_{2}\right) \in \mathbb{Z}^{2}} A_{j_{1}, j_{2}}^{k_{1}, k_{2}}(\bullet) \varepsilon_{j_{1}, j_{2}}^{k_{1}, k_{2}}(\omega)$ is normally convergent with respect to the uniform norm $\|\cdot\|_{I, \infty}$, that is one has

$$
\sum_{\left(k_{1}, k_{2}\right) \in \mathbb{Z}^{2}}\left\|A_{j_{1}, j_{2}}^{k_{1}, k_{2}}\right\|_{I, \infty}\left|\varepsilon_{j_{1}, j_{2}}^{k_{1}, k_{2}}(\omega)\right|<+\infty .
$$


Using (2.2), (2.6), (2.10), the definition of $\|\cdot\|_{I, \infty}$, and the triangle inequality, one gets, for some positive finite constant $C_{1}$ depending on $T,\left(j_{1}, j_{2}\right) \in \mathbb{Z}^{2}$ and $\omega$, that

$$
\begin{aligned}
& \sum_{\left(k_{1}, k_{2}\right) \in \mathbb{Z}^{2}}\left\|A_{j_{1}, j_{2}}^{k_{1}, k_{2}}\right\|_{I, \infty}\left|\varepsilon_{j_{1}, j_{2}}^{k_{1}, k_{2}}(\omega)\right| \\
\leq & C_{1} \sum_{\left(k_{1}, k_{2}\right) \in \mathbb{Z}^{2}} \int_{0}^{T} \frac{\sqrt{\log \left(3+\left|j_{1}\right|+\left|k_{1}\right|\right) \log \left(3+\left|j_{2}\right|+\left|k_{2}\right|\right)}}{\left(1+2^{j_{1}} T+\left|2^{j_{1}} s-k_{1}\right|\right)^{2}\left(1+2^{j_{2}} T+\left|2^{j_{2}} s-k_{2}\right|\right)^{2}} d s \\
\leq & C_{1} \sum_{\left(k_{1}, k_{2}\right) \in \mathbb{Z}^{2}} \int_{0}^{T} \frac{\sqrt{\log \left(3+\left|j_{1}\right|+\left|k_{1}\right|\right) \log \left(3+\left|j_{2}\right|+\left|k_{2}\right|\right)}}{\left(1+2^{j_{1}} T+\left|k_{1}\right|-\left|2^{j_{1}} s\right|\right)^{2}\left(1+2^{j_{2}} T+\left|k_{2}\right|-\left|2^{j_{2}} s\right|\right)^{2}} d s \\
\leq & C_{1} T \sum_{\left(k_{1}, k_{2}\right) \in \mathbb{Z}^{2}} \frac{\sqrt{\log \left(3+\left|j_{1}\right|+\left|k_{1}\right|\right) \log \left(3+\left|j_{2}\right|+\left|k_{2}\right|\right)}}{\left(1+\left|k_{1}\right|\right)^{2}\left(1+\left|k_{2}\right|\right)^{2}}<+\infty,
\end{aligned}
$$

which shows that (2.78) holds. Next, for each fixed $\left(j_{1}, j_{2}\right) \in \mathbb{Z}^{2}$, one denotes by $\left\{X_{j_{1}, j_{2}}(t)\right\}_{t \in I}$ the stochastic process with continuous paths which vanishes outside of the event $\Omega^{*}$, and which is defined on $\Omega^{*}$, for all $(t, \omega) \in I \times \Omega^{*}$, as

$$
X_{j_{1}, j_{2}}(t, \omega)=\sum_{\left(k_{1}, k_{2}\right) \in \mathbb{Z}^{2}} A_{j_{1}, j_{2}}^{k_{1}, k_{2}}(t) \varepsilon_{j_{1}, j_{2}}^{k_{1}, k_{2}}(\omega)
$$

Let us now show that in order to derive (1.20) it is enough to prove that there are $\check{\Omega}$ an event of probability 1 included in $\Omega^{*}$, and $\check{C}$ a positive finite random variable such that one has on $\check{\Omega}$, for every $J \in \mathbb{N}$,

$$
\sum_{\left(j_{1}, j_{2}\right) \in \mathbb{Z}^{2}, j_{1} \vee j_{2} \geq J} 2^{j_{1}\left(1-H_{1}\right)+j_{2}\left(1-H_{2}\right)}\left\|X_{j_{1}, j_{2}}\right\|_{I, \infty} \leq \check{C} J 2^{-J\left(H_{1}+H_{2}-3 / 2\right)} .
$$

Assuming that $(2.80)$ is true, then it turns out that, for all fixed $J \in \mathbb{N}$ and for every $\omega \in \check{\Omega}$ the series of continuous functions

$$
X_{J}(\bullet, \omega):=\sum_{\left(j_{1}, j_{2}\right) \in \mathbb{Z}^{2}, j_{1} \vee j_{2} \geq J} 2^{j_{1}\left(1-H_{1}\right)+j_{2}\left(1-H_{2}\right)} X_{j_{1}, j_{2}}(\bullet, \omega)
$$

is normally convergent with respect to the norm $\|\cdot\|_{I, \infty}$; thus, $X_{J}(\bullet, \omega)$ is a continuous function on $I$. In the sequel, one denotes by $\left\{X_{J}(t)\right\}_{t \in I}$ the stochastic process with continuous paths which vanishes outside of $\check{\Omega}$ and which is defined through (2.81) on $\check{\Omega}$. It can easily be seen that (2.80) and the triangle inequality imply, almost surely for all $J \in \mathbb{N}$, that

$$
\left\|X_{J}\right\|_{I, \infty} \leq \check{C} J 2^{-J\left(H_{1}+H_{2}-3 / 2\right)} .
$$

On the other hand, one knows from (2.79), (2.5) and (1.16), that, for all fixed $J \in \mathbb{N}$ and $t \in I$, the random series

$$
\sum_{\left(j_{1}, j_{2}\right) \in \mathbb{Z}^{2}, j_{1} \vee j_{2} \geq J} 2^{j_{1}\left(1-H_{1}\right)+j_{2}\left(1-H_{2}\right)} X_{j_{1}, j_{2}}(t)
$$


converges to $R_{H_{1}, H_{2}}(t)-R_{H_{1}, H_{2}, J}(t)$ in $L^{2}(\Omega)$. Combining the later fact with (2.81), one obtains, for all $t \in I$ almost surely,

$$
X_{J}(t)=R_{H_{1}, H_{2}}(t)-R_{H_{1}, H_{2}, J}(t) .
$$

The latter equality and the fact that the stochastic processes $\left\{R_{H_{1}, H_{2}}(t)-R_{H_{1}, H_{2}, J}(t)\right\}_{t \in I}$ and $\left\{X_{J}(t)\right\}_{t \in I}$ have continuous paths imply that they are indistinguishable. Thus, (1.20) is nothing else than (2.82).

Finally, let us show that (2.80) holds. Using (2.79), the triangle inequality, (2.20), (2.21), (2.24), (2.25), (2.28), (2.29), (2.43), (2.44), (2.51) and (2.52), one gets, for all $J \in \mathbb{N}$, that

$$
\sum_{\left(j_{1}, j_{2}\right) \in \mathbb{Z}^{2}, j_{1} \vee j_{2} \geq J} 2^{j_{1}\left(1-H_{1}\right)+j_{2}\left(1-H_{2}\right)}\left\|X_{j_{1}, j_{2}}\right\|_{I, \infty} \leq \sum_{l=1}^{2}\left(\mathcal{M}_{l, J}+\sum_{m=1}^{4} \mathcal{H}_{l, J}^{m}\right) .
$$

Therefore, combining Lemmas 2.8, 2.9 and 2.14 (in which $L$ is assumed to be large enough) with Lemmas 2.10 and 2.16, one obtains (2.80).

\section{Proof of Theorem 1.3}

In order to prove Theorem 1.3 one needs to obtain some intermediary results.

Lemma 3.1 The real numbers $T>2$ and $L \geq 3 / 2$ are arbitrary and fixed. For every $n \in \mathbb{N}$, let $\mathcal{L}_{1, n}^{1}$ and $\mathcal{L}_{2, n}^{1}$ be the positive random variables defined as:

$$
\mathcal{L}_{1, n}^{1}:=\sum_{j_{1} \vee j_{2}<n} 2^{j_{1}\left(1-H_{1}\right)+j_{2}\left(1-H_{2}\right)} \sum_{k_{1} \in \mathbb{Z}} \sum_{\left|k_{2}\right|>2^{n+1} T} \sup _{t \in[0, T]}\left\{\left|A_{j_{1}, j_{2}}^{k_{1}, k_{2}}(t)\right|\right\}\left|\varepsilon_{j_{1}, j_{2}}^{k_{1}, k_{2}}\right|
$$

and

$$
\mathcal{L}_{2, n}^{1}:=\sum_{j_{1} \vee j_{2}<n} 2^{j_{1}\left(1-H_{1}\right)+j_{2}\left(1-H_{2}\right)} \sum_{\left|k_{1}\right|>2^{n+1} T} \sum_{k_{2} \in \mathbb{Z}} \sup _{t \in[0, T]}\left\{\left|A_{j_{1}, j_{2}}^{k_{1}, k_{2}}(t)\right|\right\}\left|\varepsilon_{j_{1}, j_{2}}^{k_{1}, k_{2}}\right| .
$$

Then, there exists an almost surely finite random variable $C$ such that, for all $n \in \mathbb{N}$ and $l \in\{1,2\}$, the following inequality holds on the event $\Omega^{*}$ of probability 1 which was introduced in Lemma 2.4:

$$
\mathcal{L}_{l, n}^{1} \leq C 2^{-n\left(H_{1}+H_{2}+L-3\right)} n \log (3+n) .
$$


Proof of Lemma 3.1 One only shows that (3.3) is satisfied when $l=1$, the case where $l=2$ can be treated in the same way. Let $n \in \mathbb{N}$ and $\left(j_{1}, j_{2}\right) \in \mathbb{Z}^{2}$ be arbitrary, fixed and such that

$$
j_{1} \vee j_{2}<n \text {. }
$$

Using (2.6), (2.2), (2.10), (4.1), the triangle inequality, (3.4), Lemma 4.2, the fact that $y \mapsto(2+y)^{-L} \sqrt{\log (2+y)}$ is a decreasing function on $\mathbb{R}_{+}$, and (4.2), one gets that

$$
\begin{aligned}
& \sum_{k_{1} \in \mathbb{Z}} \sum_{\left|k_{2}\right|>2^{n+1} T} \sup _{t \in[0, T]}\left\{\left|A_{j_{1}, j_{2}}^{k_{1}, k_{2}}(t)\right|\right\}\left|\varepsilon_{j_{1}, j_{2}}^{k_{1}, k_{2}}\right| \\
& \leq C_{0} \sum_{k_{1} \in \mathbb{Z}} \sum_{\left|k_{2}\right|>2^{n+1} T} \int_{0}^{T} \frac{\sqrt{\log \left(3+\left|j_{1}\right|+\left|k_{1}\right|\right)}}{\left(3+\left|2^{j_{1}} s-k_{1}\right|\right)^{L}} \times \frac{\sqrt{\log \left(3+\left|j_{2}\right|+\left|k_{2}\right|\right)}}{\left(3+\left|2^{j_{2}} s-k_{2}\right|\right)^{L}} d s \\
& \leq C_{0} \int_{0}^{T}\left(\sum_{k_{1} \in \mathbb{Z}} \sum_{\left|k_{2}\right|>2^{n+1} T} \frac{\sqrt{\log \left(3+\left|j_{1}\right|+\left|k_{1}\right|\right)}}{\left(3+\left|2^{j_{1}} s-k_{1}\right|\right)^{L}} \times \frac{\sqrt{\log \left(3+\left|j_{2}\right|+\left|k_{2}\right|\right)}}{\left(3+\left|k_{2}\right|-2^{j_{2}} s\right)^{L}}\right) d s \\
& \leq C_{1} T 2^{L} \sqrt{\log \left(3+\left|j_{1}\right|+2^{j_{1}} T\right) \log \left(3+\left|j_{2}\right|\right)} \sum_{2^{n+1} T} \frac{\sqrt{\log \left(3+\left|k_{2}\right|\right)}}{\left(3+\left|k_{2}\right|\right)^{L}} \\
& \leq C_{1} T 2^{L+1} \sqrt{\log \left(3+\left|j_{1}\right|+2^{j_{1}} T\right) \log \left(3+\left|j_{2}\right|\right)} \int_{2^{n+1} T}^{+\infty} \frac{\sqrt{\log (2+y)}}{(2+y)^{L}} d y \\
& \leq C_{2} \sqrt{\log \left(3+\left|j_{1}\right|\right) \log \left(3+\left|j_{2}\right|\right)} n 2^{-n(L-1)},
\end{aligned}
$$

where $C_{0}, C_{1}$ and $C_{2}$ are 3 positive finite random constants not depending on $\left(j_{1}, j_{2}\right)$ and $n$. Next, it follows from (3.1) and (3.5) that

$$
\begin{aligned}
\mathcal{L}_{1, n}^{1} & \leq C_{2} n 2^{-n(L-1)} \sum_{j_{1}=-\infty}^{n-1} \sum_{j_{2}=-\infty}^{n-1} 2^{j_{1}\left(1-H_{1}\right)+j_{2}\left(1-H_{2}\right)} \sqrt{\log \left(3+\left|j_{1}\right|\right) \log \left(3+\left|j_{2}\right|\right)} \\
& \leq C_{3} 2^{-n\left(H_{1}+H_{2}+L-3\right)} n \log (3+n),
\end{aligned}
$$

where $C_{3}$ is a positive finite random constant not depending on $n$. Thus, one obtains (3.3) when $l=1$.

Lemma 3.2 The real numbers $T>2, L>1$ and $g>0$ are arbitrary and fixed. For every $n \in \mathbb{N}$, let $\mathcal{L}_{1, n}^{2}$ and $\mathcal{L}_{2, n}^{2}$ be the positive random variables defined as:

$$
\mathcal{L}_{1, n}^{2}:=\sum_{\left(j_{1}, j_{2}\right) \in \mathbb{N}^{2}} 2^{-j_{1}\left(1-H_{1}\right)-j_{2}\left(1-H_{2}\right)} \sum_{k_{1} \in \mathbb{Z}} \sum_{\left|k_{2}\right|>2^{n g}} \sup _{t \in[0, T]}\left\{\left|A_{-j_{1},-j_{2}}^{k_{1}, k_{2}}(t)\right|\right\}\left|\varepsilon_{-j_{1},-j_{2}}^{k_{1}, k_{2}}\right|
$$

and

$$
\mathcal{L}_{2, n}^{2}:=\sum_{\left(j_{1}, j_{2}\right) \in \mathbb{N}^{2}} 2^{-j_{1}\left(1-H_{1}\right)-j_{2}\left(1-H_{2}\right)} \sum_{\left|k_{1}\right|>2^{n g}} \sum_{k_{2} \in \mathbb{Z}} \sup _{t \in[0, T]}\left\{\left|A_{-j_{1},-j_{2}}^{k_{1}, k_{2}}(t)\right|\right\}\left|\varepsilon_{-j_{1},-j_{2}}^{k_{1}, k_{2}}\right| .
$$


Then, there exists an almost surely finite random variable $C$ such that, for all $n \in \mathbb{N}$ and $l \in\{1,2\}$, the following inequality holds on the event $\Omega^{*}$ :

$$
\mathcal{L}_{l, n}^{2} \leq C 2^{-n(L-1) g} \sqrt{n} .
$$

Proof of Lemma 3.2 One only shows that (3.8) is satisfied when $l=1$, the case where $l=2$ can be treated in the same way. Let $n \in \mathbb{N}$ and $\left(j_{1}, j_{2}\right) \in \mathbb{N}^{2}$ be arbitrary and fixed. Using (2.6), (2.2), (2.10), (4.1), the triangle inequality, and the fact that $y \mapsto(2+y)^{-L} \sqrt{\log (2+y)}$ is a decreasing function on $\mathbb{R}_{+}$, one gets that

$$
\begin{aligned}
& \sum_{k_{1} \in \mathbb{Z}} \sum_{\left|k_{2}\right|>2^{n g}} \sup _{t \in[0, T]}\left\{\left|A_{-j_{1},-j_{2}}^{k_{1}, k_{2}}(t)\right|\right\}\left|\varepsilon_{-j_{1},-j_{2}}^{k_{1}, k_{2}}\right| \\
& \leq C_{0} \sum_{k_{1} \in \mathbb{Z}} \sum_{\left|k_{2}\right|>2^{n g}} \int_{0}^{T} \frac{\sqrt{\log \left(3+j_{1}+\left|k_{1}\right|\right)}}{\left(3+T+\left|2^{-j_{1}} s-k_{1}\right|\right)^{L}} \times \frac{\sqrt{\log \left(3+j_{2}+\left|k_{2}\right|\right)}}{\left(3+T+\left|2^{-j_{2}} s-k_{2}\right|\right)^{L}} d s \\
& \leq C_{0} \sqrt{\log \left(3+j_{1}\right) \log \left(3+j_{2}\right)} \int_{0}^{T}\left(\sum_{k_{1} \in \mathbb{Z}\left|k_{2}\right|>2^{n g}} \frac{\sqrt{\log \left(3+\left|k_{1}\right|\right)}}{\left(3+\left|k_{1}\right|\right)^{L}} \times \frac{\sqrt{\log \left(3+\left|k_{2}\right|\right)}}{\left(3+\left|k_{2}\right|\right)^{L}}\right) d s \\
& \leq C_{1} \sqrt{\log \left(3+j_{1}\right) \log \left(3+j_{2}\right)} \sum_{\left|k_{2}\right|>2^{n g}} \frac{\sqrt{\log \left(3+\left|k_{2}\right|\right)}}{\left(3+\left|k_{2}\right|\right)^{L}} \\
& \leq C_{1} \sqrt{\log \left(3+j_{1}\right) \log \left(3+j_{2}\right)} \int_{2^{n g}}^{+\infty} \frac{\sqrt{\log (2+y)}}{(2+y)^{L}} d y \\
& \leq C_{2} \sqrt{\log \left(3+j_{1}\right) \log \left(3+j_{2}\right)} 2^{-n(L-1) g} \sqrt{n}
\end{aligned}
$$

where $C_{0}, C_{1}$ and $C_{2}$ are 3 positive finite random constants not depending on $\left(j_{1}, j_{2}\right)$ and $n$. Next, it follows from (3.6) and (3.9) that

$$
\begin{aligned}
\mathcal{L}_{1, n}^{2} & \leq C_{2} 2^{-n(L-1) g} \sqrt{n} \sum_{\left(j_{1}, j_{2}\right) \in \mathbb{N}^{2}} 2^{-j_{1}\left(1-H_{1}\right)-j_{2}\left(1-H_{2}\right)} \sqrt{\log \left(3+j_{1}\right) \log \left(3+j_{2}\right)} \\
& =C_{3} 2^{-n(L-1) g} \sqrt{n},
\end{aligned}
$$

where $C_{3}$ is a positive finite random constant not depending on $n$. Thus, one obtains (3.8) when $l=1$.

Lemma 3.3 The real numbers $T>2$ and $b>0$ are arbitrary and fixed. For every $n \in \mathbb{N}$, let $\mathcal{L}_{1, n}^{3}$ and $\mathcal{L}_{2, n}^{3}$ be the positive random variables defined as:

$$
\mathcal{L}_{1, n}^{3}:=\sum_{j_{1}=0}^{n-1} \sum_{j_{2}>2^{n b}} 2^{j_{1}\left(1-H_{1}\right)-j_{2}\left(1-H_{2}\right)} \sum_{k_{1} \in \mathbb{Z}} \sum_{k_{2} \in \mathbb{Z}} \sup _{t \in[0, T]}\left\{\left|A_{j_{1},-j_{2}}^{k_{1}, k_{2}}(t)\right|\right\}\left|\varepsilon_{j_{1},-j_{2}}^{k_{1}, k_{2}}\right|
$$


and

$$
\mathcal{L}_{2, n}^{3}:=\sum_{j_{1}>2^{n b}} \sum_{j_{2}=0}^{n-1} 2^{-j_{1}\left(1-H_{1}\right)+j_{2}\left(1-H_{2}\right)} \sum_{k_{1} \in \mathbb{Z}} \sum_{k_{2} \in \mathbb{Z}} \sup _{t \in[0, T]}\left\{\left|A_{-j_{1}, j_{2}}^{k_{1}, k_{2}}(t)\right|\right\}\left|\varepsilon_{-j_{1}, j_{2}}^{k_{1}, k_{2}}\right| .
$$

Then, there exists an almost surely finite random variable $C$ such that, for all $n \in \mathbb{N}$ and $l \in\{1,2\}$, the following inequality holds on the event $\Omega^{*}$ :

$$
\mathcal{L}_{l, n}^{3} \leq C n 2^{\left(1-H_{1} \wedge H_{2}\right) n-\left(1-H_{1} \vee H_{2}\right) 2^{n b}} .
$$

Proof of Lemma 3.3 One only shows that (3.12) is satisfied when $l=1$, the case where $l=2$ can be treated in the same way. Let $n \in \mathbb{N}$ and $\left(j_{1}, j_{2}\right) \in \mathbb{Z}_{+}^{2}$ be arbitrary, fixed and such that

$$
0 \leq j_{1}<n \quad \text { and } \quad j_{2}>2^{n b}
$$

Using (2.6), (2.2), (2.10), (4.1), the triangle inequality, (3.13), Lemma 4.2 and (4.2), one gets that

$$
\begin{aligned}
& \sum_{k_{1} \in \mathbb{Z}} \sum_{k_{2} \in \mathbb{Z}} \sup _{t \in[0, T]}\left\{\left|A_{j_{1},-j_{2}}^{k_{1}, k_{2}}(t)\right|\right\}\left|\varepsilon_{j_{1},-j_{2}}^{k_{1}, k_{2}}\right| \\
& \leq C_{0} \sum_{k_{1} \in \mathbb{Z}} \sum_{k_{2} \in \mathbb{Z}} \int_{0}^{T} \frac{\sqrt{\log \left(3+j_{1}+\left|k_{1}\right|\right)}}{\left(3+\left|2^{j_{1}} s-k_{1}\right|\right)^{2}} \times \frac{\sqrt{\log \left(3+j_{2}+\left|k_{2}\right|\right)}}{\left(3+T+\left|2^{-j_{2}} s-k_{2}\right|\right)^{2}} d s \\
& \leq C_{0} \sqrt{\log \left(3+j_{2}\right)} \int_{0}^{T}\left(\sqrt{\log \left(3+j_{1}+2^{j_{1}} T\right)} \sum_{k_{2} \in \mathbb{Z}} \frac{\sqrt{\log \left(3+\left|k_{2}\right|\right)}}{\left(3+\left|k_{2}\right|\right)^{2}}\right) d s \\
& \leq C_{1} \sqrt{n \log \left(3+j_{2}\right)},
\end{aligned}
$$

where $C_{0}$ and $C_{1}$ are 2 positive finite random constants not depending on $\left(j_{1}, j_{2}\right)$ and $n$. Next, it follows from (3.10) and (3.14) that

$$
\begin{aligned}
\mathcal{L}_{1, n}^{3} & \leq C_{1} \sqrt{n} \sum_{j_{1}=0}^{n-1} \sum_{j_{2}>2^{n b}} 2^{j_{1}\left(1-H_{1}\right)-j_{2}\left(1-H_{2}\right)} \sqrt{\log \left(3+j_{2}\right)} \\
& \leq C_{2} n 2^{\left(1-H_{1}\right) n-\left(1-H_{2}\right) 2^{n b}} \leq C_{2} n 2^{\left(1-H_{1} \wedge H_{2}\right) n-\left(1-H_{1} \vee H_{2}\right) 2^{n b}},
\end{aligned}
$$

where $C_{2}$ is a positive finite random constant not depending on $n$. Thus, one obtains (3.12) when $l=1$. 
Lemma 3.4 The real numbers $T>2$ and $d>0$ are arbitrary and fixed. For every $n \in \mathbb{N}$, let $\mathcal{Q}_{n}$ be the positive random variable defined as:

$$
\mathcal{Q}_{n}:=\sum_{\left(j_{1}, j_{2}\right) \in \mathbb{N}^{2}, j_{1} \vee j_{2}>2^{\text {nd }}} 2^{-j_{1}\left(1-H_{1}\right)-j_{2}\left(1-H_{2}\right)} \sum_{k_{1} \in \mathbb{Z}} \sum_{k_{2} \in \mathbb{Z}} \sup _{t \in[0, T]}\left\{\left|A_{-j_{1},-j_{2}}^{k_{1}, k_{2}}(t)\right|\right\}\left|\varepsilon_{-j_{1},-j_{2}}^{k_{1}, k_{2}}\right| .
$$

Then, there exists an almost surely finite random variable $C$ such that, for all $n \in \mathbb{N}$, the following inequality holds on the event $\Omega^{*}$ :

$$
\mathcal{Q}_{n} \leq C 2^{-\left(1-H_{1} \vee H_{2}\right) 2^{n d}} \sqrt{n} .
$$

Proof of Lemma 3.4 Let $\left(j_{1}, j_{2}\right) \in \mathbb{N}^{2}$ be arbitrary and fixed. Using (2.6), (2.2), (2.10), (4.1) and the triangle inequality, one gets that

$$
\begin{aligned}
& \sum_{k_{1} \in \mathbb{Z}} \sum_{k_{2} \in \mathbb{Z}} \sup _{t \in[0, T]}\left\{\left|A_{-j_{1},-j_{2}}^{k_{1}, k_{2}}(t)\right|\right\}\left|\varepsilon_{-j_{1},-j_{2}}^{k_{1}, k_{2}}\right| \\
& \leq C_{0} \sum_{k_{1} \in \mathbb{Z}} \sum_{k_{2} \in \mathbb{Z}} \int_{0}^{T} \frac{\sqrt{\log \left(3+j_{1}+\left|k_{1}\right|\right)}}{\left(3+T+\left|2^{-j_{1}} s-k_{1}\right|\right)^{2}} \times \frac{\sqrt{\log \left(3+j_{2}+\left|k_{2}\right|\right)}}{\left(3+T+\left|2^{-j_{2}} s-k_{2}\right|\right)^{2}} d s \\
& \leq C_{0} \sqrt{\log \left(3+j_{1}\right) \log \left(3+j_{2}\right)} \int_{0}^{T}\left(\sum_{k_{1} \in \mathbb{Z}} \sum_{k_{2} \in \mathbb{Z}} \frac{\sqrt{\log \left(3+\left|k_{1}\right|\right)}}{\left(3+\left|k_{1}\right|\right)^{2}} \times \frac{\sqrt{\log \left(3+\left|k_{2}\right|\right)}}{\left(3+\left|k_{2}\right|\right)^{2}}\right) d s \\
& =C_{1} \sqrt{\log \left(3+j_{1}\right) \log \left(3+j_{2}\right)},
\end{aligned}
$$

where $C_{0}$ and $C_{1}$ are 2 positive finite random constants not depending on $\left(j_{1}, j_{2}\right)$. Next, it follows from (3.15) and (3.17) that, for all $n \in \mathbb{N}$, one has

$$
\begin{aligned}
\mathcal{Q}_{n} & \leq C_{1} \sum_{\left(j_{1}, j_{2}\right) \in \mathbb{N}^{2}, j_{1} \vee j_{2}>2^{\text {nd }}} 2^{-j_{1}\left(1-H_{1}\right)-j_{2}\left(1-H_{2}\right)} \sqrt{\log \left(3+j_{1}\right) \log \left(3+j_{2}\right)} \\
& \leq C_{2} 2^{-\left(1-H_{1} \vee H_{2}\right) 2^{\text {nd }}} \sqrt{n},
\end{aligned}
$$

where $C_{2}$ is a positive finite random constant not depending on $n$. Thus, one obtains (3.16).

We are now in position to complete the prove Theorem 1.3.

End of the proof of Theorem 1.3 Let $\Omega^{*}$ and $\Omega^{* *}$ be the same events of probability 1 as in Lemmas 2.4 and 2.16. Let us first show that in order to derive (1.25) it is enough to prove that there exists an almost surely finite random variable $C$ such that the inequality

$$
\left\|\widetilde{R}_{H_{1}, H_{2}, n+p}-\widetilde{R}_{H_{1}, H_{2}, n}\right\|_{I, \infty} \leq C n 2^{-n\left(H_{1}+H_{2}-3 / 2\right)}
$$


holds, for all $(n, p) \in \mathbb{N}^{2}$, on the event $\Omega^{*} \cap \Omega^{* *}$ of probability 1. Assuming that (3.18) is true, then it turns out that, for every fixed $\omega \in \Omega^{*} \cap \Omega^{* *}$, the sequence of continuous functions $\left(\widetilde{R}_{H_{1}, H_{2}, n}(\bullet, \omega)\right)_{n \in \mathbb{N}}$ is a Cauchy sequence in the Banach space of the continuous functions over $I$ equipped with the norm $\|\cdot\|_{I, \infty}$. Thus, for any fixed $\omega \in \Omega^{*} \cap \Omega^{* *}$, it converges to a continuous function over $I$ denoted by $\widetilde{R}_{H_{1}, H_{2}}(\bullet, \omega)$. On the other hand, when $\omega \notin \Omega^{*} \cap \Omega^{* *}$, one assumes that $\widetilde{R}_{H_{1}, H_{2}}(\bullet, \omega)$ is the vanishing function. Next observe that one has

$$
\widetilde{R}_{H_{1}, H_{2}}(t)=R_{H_{1}, H_{2}}(t), \quad \text { for all } t \in I, \text { almost surely, }
$$

since one knows from (1.21) and (1.22) that, for any fixed $t \in I$, the sequence of random variables $\left(\widetilde{R}_{H_{1}, H_{2}, n}(t)\right)_{n \in \mathbb{N}}$ converges to $R_{H_{1}, H_{2}}(t)$ in $L^{2}(\Omega)$. The equality (3.19) implies that the stochastic processes $\left\{\widetilde{R}_{H_{1}, H_{2}}(t)\right\}_{t \in I}$ and $\left\{R_{H_{1}, H_{2}}(t)\right\}_{t \in I}$ are indistinguishable since they have continuous paths. Thus, letting $p$ in (3.18) tend to $+\infty$, one obtains (1.25).

Finally, let us show that (3.18) holds. Using (1.22), (1.23), (1.24), the equality $I:=[0, T]$, (2.12), the inclusion

$$
D_{j}^{1}(t) \subset\left\{k \in \mathbb{Z}:|k| \leq 2^{n+p+1} T\right\}, \quad \text { for all } t \in I \text { and }(j, n, p) \in \mathbb{N}^{3} \text { s.t. } n<j \leq n+p,
$$

the triangle inequality, (2.20), (2.21), (2.24), (2.25), (2.28), (2.29), (2.43), (2.44), (2.51), (2.52), (3.1), (3.2), (3.6), (3.7), (3.10), (3.11), and (3.15), one gets, for all $(n, p) \in \mathbb{N}^{2}$, that

$$
\left\|\widetilde{R}_{H_{1}, H_{2}, n+p}-\widetilde{R}_{H_{1}, H_{2}, n}\right\|_{I, \infty} \leq \mathcal{Q}_{n}+\sum_{l=1}^{2}\left(\mathcal{M}_{l, n}+\sum_{m=1}^{4} \mathcal{H}_{l, n}^{m}+\sum_{m^{\prime}=1}^{3} \mathcal{L}_{l, n}^{m^{\prime}}\right) .
$$

Therefore, combining Lemmas 2.8, 2.9, 2.14, 3.1, and 3.2 (in which $L$ is assumed to be large enough) with Lemmas 2.10, 2.16, 3.3, and 3.4, one obtains (3.18).

\section{Appendix}

Lemma 4.1 For every $(x, y) \in \mathbb{R}_{+}^{2}$, one has

$$
\log (3+x+y) \leq \log (3+x) \log (3+y) .
$$

Moreover, for any fixed positive real number $T$ there exists a constant $c>0$ such that, for all $x \in \mathbb{R}_{+}$, the following inequality holds:

$$
\log \left(3+x+2^{x} T\right) \leq c(1+x) .
$$

The proof of Lemma 4.1 is standard and easy this is why it has been omitted. 
Lemma 4.2 For any fixed real number $L>1$, there exists a constant $c>0$ such that, for all $j \in \mathbb{Z}$ and for each $s \in \mathbb{R}$, one has:

$$
\sum_{k \in \mathbb{Z}} \frac{\sqrt{\log (3+|j|+|k|)}}{\left(3+\left|2^{j} s-k\right|\right)^{L}} \leq c \sqrt{\log \left(3+|j|+2^{j}|s|\right)} .
$$

Proof of Lemma 4.2 Setting $m=k-\left\lfloor 2^{j} s\right\rfloor$, where $\left\lfloor 2^{j} s\right\rfloor$ denotes the integer part of $2^{j} s$, and using the triangle inequality and (4.1), one obtains that

$$
\begin{aligned}
\sum_{k \in \mathbb{Z}} \frac{\sqrt{\log (3+|j|+|k|)}}{\left(3+\left|2^{j} s-k\right|\right)^{L}} & =\sum_{m \in \mathbb{Z}} \frac{\sqrt{\log \left(3+|j|+\mid m+\left\lfloor 2^{j} s\right\rfloor\right)}}{\left(3+\left|2^{j} s-\left\lfloor 2^{j} s\right\rfloor-m\right|\right)^{L}} \\
& \leq \sqrt{\log \left(3+|j|+2^{j}|s|\right)} \sum_{m \in \mathbb{Z}} \frac{\sqrt{\log (4+|m|)}}{\left(3+\left|2^{j} s-\left\lfloor 2^{j} s\right\rfloor-m\right|\right)^{L}}
\end{aligned}
$$

Then, noticing that

$$
3+\left|2^{j} s-\left\lfloor 2^{j} s\right\rfloor-m\right| \geq 2+|m|
$$

one gets that

$$
\sum_{k \in \mathbb{Z}} \frac{\sqrt{\log (3+|j|+|k|)}}{\left(3+\left|2^{j} s-k\right|\right)^{L}} \leq c \sqrt{\log \left(3+|j|+2^{j}|s|\right)},
$$

where the constant

$$
c:=\sum_{m \in \mathbb{Z}} \frac{\sqrt{\log (4+|m|)}}{(2+|m|)^{L}}<+\infty
$$

Lemma 4.3 For each fixed real number $L>1$, there exists a constant $c>0$ such that, for every $t \in \mathbb{R}_{+}$, for all $s \in[0, t]$ and for any $j \in \mathbb{Z}_{+}$, one has

$$
\sum_{k \in D_{j}^{3}(t)} \frac{\sqrt{\log (3+j+|k|)}}{\left(3+\left|2^{j} s-k\right|\right)^{L}} \leq c(j+1) 2^{-j(L-1)(1-a)} \sqrt{\log (3+t)},
$$

where $D_{j}^{3}(t)$ is defined through (2.14) and (2.11).

Proof of Lemma 4.3 In view of (2.14) and (2.11), one has $D_{j}^{3}(t)=D_{j}^{3,+}(t) \cup D_{j}^{3,-}(t)$, where $D_{j}^{3,+}(t)$ and $D_{j}^{3,-}(t)$ are the two disjoint sets defined as:

$$
D_{j}^{3,+}(t)=\left\{k \in \mathbb{Z}, k>2^{j} t+2^{j(1-a)}\right\}
$$


and

$$
D_{j}^{3,-}(t)=\left\{k \in \mathbb{Z}, k<-2^{j(1-a)}\right\}
$$

Thus, one gets that

$$
\sum_{k \in D_{j}^{3}(t)} \frac{\sqrt{\log (3+j+|k|)}}{\left(3+\left|2^{j} s-k\right|\right)^{L}}=\sum_{k>2^{j} t+2^{j(1-a)}} \frac{\sqrt{\log (3+j+k)}}{\left(3+\left|2^{j} s-k\right|\right)^{L}}+\sum_{k<-2^{j(1-a)}} \frac{\sqrt{\log (3+j+|k|)}}{\left(3+\left|2^{j} s-k\right|\right)^{L}} .
$$

Let us now provide an appropriate upper bound for the first term in the right-hand side of (4.5). One denotes by $\lfloor\cdot\rfloor$ the integer part function. Using the change of variable $m=k-\left\lfloor 2^{j} t\right\rfloor$, the triangle inequality, (4.1), the inequality $\left\lfloor 2^{j} t\right\rfloor-2^{j} s>-1$, the inequality $\log (3+m) \leq 2+m$, and the fact that $x \mapsto(1+x)^{-L} \sqrt{\log (2+x)}$ is a decreasing function on $\mathbb{R}_{+}$, one obtains that

$$
\begin{aligned}
\sum_{k>2^{j^{j}+2^{j(1-a)}}} \frac{\sqrt{\log (3+j+k)}}{\left(3+\left|2^{j} s-k\right|\right)^{L}} & =\sum_{k>2^{j} t+2^{j(1-a)}} \frac{\sqrt{\log (3+j+k)}}{\left(3+k-2^{j} s\right)^{L}} \\
& =\sum_{m>2^{j} t-\left\lfloor 2^{j} t\right\rfloor+2^{j(1-a)}} \frac{\sqrt{\log \left(3+j+\left\lfloor 2^{j} t\right\rfloor+|m|\right)}}{\left(3+\left\lfloor 2^{j} t\right\rfloor-2^{j} s+m\right)^{L}} \\
& \leq \sqrt{\log \left(3+j+2^{j} t\right)} \sum_{m>2^{j(1-a)}} \frac{\sqrt{\log (3+m)}}{(2+m)^{L}} \\
& \leq \sqrt{\log \left(3+j+2^{j} t\right)} \int_{2^{j(1-a)}}^{+\infty} \frac{\sqrt{\log (2+x)}}{(1+x)^{L}} d x \\
& \leq c_{1} \sqrt{(j+1) \log (3+t)} 2^{-j(L-1)(1-a)} \sqrt{\log \left(2+2^{j(1-a)}\right)} \\
& \leq c_{2}(j+1) 2^{-j(L-1)(1-a)} \sqrt{\log (3+t)},
\end{aligned}
$$

where $c_{1}$ and $c_{2}$ are two positive finite constants not depending on $j, t, s$ and $a$. Similarly to (4.6), it can be shown that

$$
\sum_{k<-2^{j(1-a)}} \frac{\sqrt{\log (3+j+|k|)}}{\left(3+\left|2^{j} s-k\right|\right)^{L}} \leq c_{3} 2^{-j(L-1)(1-a)} \sqrt{(j+1) \log (3+j)},
$$

where $c_{3}$ is a positive finite constant not depending on $j, t, s$ and $a$. Finally, putting together (4.5), (4.6), (4.7) and (4.2), it follows that (4.4) holds.

\section{Acknowledgements}

This work has been partially supported by the Labex CEMPI (ANR-11-LABX-0007-01) and the GDR 3475 (Analyse Multifractale). 


\section{References}

[1] P. Abry and V. Pipiras. Wavelet-based synthesis of the Rosenblatt process. Signal Processing, 86:2326-2339, 2006.

[2] B. Arras. A white noise approach to stochastic integration with respect to the Rosenblatt process. Potential Analysis, 43(4):547-591, 2015.

[3] A. Ayache and M.S. Taqqu. Rate optimality of wavelet series approximations of fractional Brownian motion. Journal of Fourier Analysis and Applications, 9(5):451-471, 2003.

[4] S. Bai and M.S. Taqqu. Generalized Hermite processes, discrete chaos and limit theorems. Stochastic Processes and their Applications, 124(4):1710-1739, 2014.

[5] S. Bai and M.S. Taqqu. Structure of the third moment of the generalized Rosenblatt distribution. Statistics and Probability Letters, 94:144-152, 2014.

[6] S. Bai and M.S. Taqqu. Convergence of long-memory discrete k-th order Volterra processes. Stochastic Processes and their Applications, 125(5):2026-2053, 2015.

[7] S. Bai and M.S. Taqqu. Behavior of the generalized Rosenblatt process at extreme critical exponent values. The Annals of Probability, 25(2):1278-1324, 2017.

[8] S. Bai and M.S. Taqqu. The impact of the diagonals of polynomial forms on limit theorems with long memory. Bernoulli, 23(1):710-742, 2017.

[9] J.-M. Bardet and C.A. Tudor. A wavelet analysis of the Rosenblatt process: chaos expansion and estimation of the self-similarity parameter. Stochastic Processes and their Applications, 120(12):2331-2362, 2010.

[10] T. Bojdecki, L.G. Gorostiza, and A. Talarczyk. From intersection local time to the Rosenblatt process. Journal of Theoretical Probability, 28:1227-1249, 2015.

[11] I. Daubechies. Ten lectures on wavelets. Society for Industrial and Applied Mathematics, Philadelphia, PA, USA, 1992.

[12] R.L. Dobrushin and P. Major. Non-central limit theorems for non-linear functional of Gaussian fields. Zeitschrift für Wahrscheinlichkeitstheorie und Verwandte Gebiete, 50(1):22-52, 1979.

[13] P. Embrechts and M. Maejima. Self-similar processes. Academic Press, 2003. 
[14] S. Janson. Gaussian Hilbert spaces. Cambridge Tracts in Mathematics. Cambridge University Press, 1997.

[15] M. Maejima and C.A. Tudor. Selfsimilar processes with stationary increments in the second Wiener chaos. Probability and Mathematical Statistics, 32(1):167-186, 2012.

[16] M. Maejima and C.A. Tudor. On the distribution of the Rosenblatt process. Statistics and Probability Letters, 83(6):1490-1495, 2013.

[17] Y. Meyer. Wavelets and operators, volume 37. Cambridge University Press, 1992.

[18] D. Nualart. The Malliavin calculus and related topics. Number 2. Springer-Verlag Berlin Heidelberg, 2006.

[19] V. Pipiras. Wavelet-type expansion of the Rosenblatt process. Journal of Fourier Analysis and Applications, 10(6):599-634, 2004.

[20] M. Rosenblatt. Independence and dependence. In Proc. 4th Berkeley Symp. Math. Stat. Probab., volume 2, pages 431-443. University California Press, Berkeley, CA, 1961.

[21] S.G. Samko, A.A. Kilbas, and O.I Marichev. Fractional integrals and derivatives: theory and applications. Gordon and Breach Science Publishers, 1993.

[22] G. Samorodnitsky and M.S. Taqqu. Stable non-Gaussian random variables: stochastic models with infinite variance. Chapman and Hall, New York, 1994.

[23] M.S. Taqqu. Weak convergence to fractional Brownian motion and to the Rosenblatt process. Probability Theory and Related Fields, 31(4):287-302, 1975.

[24] M.S. Taqqu. Convergence of integrated processes of arbitrary Hermite rank. Zeitschrift für Wahrscheinlichkeitstheorie und Verwandte Gebiete, 50(1):53-83, 1979.

[25] M.S. Veillette and M.S. Taqqu. Properties and numerical evaluation of the Rosenblatt distribution. Bernoulli, 19(3):982-1005, 2013. 